Supplement of Earth Syst. Sci. Data, 13, 953-968, 2021

https://doi.org/10.5194/essd-13-953-2021-supplement

(c) Author(s) 2021. This work is distributed under

the Creative Commons Attribution 4.0 License.

(c) (1)

Supplement of

\title{
A standardized database of Marine Isotope Stage 5e sea-level proxies in southern Africa (Angola, Namibia and South Africa)
}

\author{
J. Andrew G. Cooper and Andrew N. Green \\ Correspondence to: J. Andrew G. Cooper (jag.cooper@ulster.ac.uk)
}

The copyright of individual parts of the supplement might differ from the CC BY 4.0 License. 
This file was exported from WALIS on 21012021

The data in this file were compiled in WALIS, the World Atlas of Last Interglacial Shorelines. WALIS is a product of the $\mathrm{E}$

The sheets in this file contain the following information (if available):

- Summary RSL datapoints: RSL datapoints from stratigraphic records, U-Series from corals and U-Series from speleotr - RSL proxies: RSL indicators from stratigraphic information, with grouped age details

- RSL indicators: list of types of RSL indicators used in the 'RSL proxies' sheet

- Elevation measurement techniques: list of el evation survey methods used both in the 'RSL proxies' sheet and in the e - Geographic positioning: list of geographic positioning methods used in the 'RSL proxies' sheet

- Sea level datums: list of datums used both in the 'RSL proxies' sheet and in the datums fields of dated samples

- U-Series (mollusks): list of all the mollusks (or algae) samples dated with U-Series. This sheet contains all U-Series mol proxies' sheet and all the U-Series mollusk/algal ages used as age banchmarks to AAR ages contained in the 'RSL proxies - Amino Acid Racemization: list of all the samples dated with AAR. This sheet contains all AAR ages created by the user , - Luminescence: list of all the samples dated with luminescence. This sheet contains all luminescence ages created by 1 luminescence ages used as age banchmarks to AAR ages contained in the 'RSL proxies tab'

- Chronostratigraphy: list of all the chronostratigraphic age constraints. This sheet contains all constraints created by the chronostratigraphic constraints ages used as age banchmarks to AAR ages contained in the 'RSL proxies tab'

- References: list of all references contained in the culled database.

Information on each field can be found at: https://walis-help.readthedocs.io/en/latest/

Information on WALIS (Including data download) can be found at: https://warmcoasts.eu/world-atlas.html

Suggested acknowledgments: The data used in this study were [extracted from / compiled in] WALIS, a sea-level databc INQUA) working group. The database structure was designed by A. Rovere, D. Ryan, T. Lorscheid, A. Dutton, P. Chutcha contributed to WALIS by: Andrew Cooper, WALIS Admin, Alessio Rovere, Ann-Kathrin Petersen, Deirdre Ryan (in order 


\begin{tabular}{|c|c|c|}
\hline WALIS_ID & Latitude & Longitude \\
\hline RSL_370 & -12.620277 & 13.243611 \\
\hline RSL_371 & -12.620277 & 13.243611 \\
\hline RSL_372 & -15.133055 & 12.154722 \\
\hline RSL_373 & -12.61 & 13.365 \\
\hline RSL_373 & -12.61 & 13.365 \\
\hline RSL_373 & -12.61 & 13.365 \\
\hline RSL_373 & -12.61 & 13.365 \\
\hline RSL_377 & -12.550833 & 13.439722 \\
\hline RSL_377 & -12.550833 & 13.439722 \\
\hline RSL_379 & -12.544166 & 13.463888 \\
\hline RSL_3435 & -33.006137 & 27.925226 \\
\hline RSL_3436 & -33.006137 & 27.925226 \\
\hline RSL_3437 & -34.205183 & 22.049697 \\
\hline RSL_3438 & -34.205183 & 22.049697 \\
\hline
\end{tabular}


RSL_3439

$-34.205183$

22.049697

RSL_356

$-34.0325$

22.7978

RSL_358

$-34.0325$

22.7978

RSL_359

$-34.056111$

22.240555

RSL_360

$-34.81722$

20.0275

RSL_361

$-34.0325$

22.7977

RSL_362

$-34.0325$

22.7977

RSL_363

$-34.0325$

22.7978

RSL_364

$-34.056111$

22.24055

RSL_365

$-32.994722$

27.95

RSL_366

$-33.006027$

27.93131

RSL_367

$-33.0225$

27.9311

RSL_368

$-27.50777$

32.6975

RSL_369

$-27.50777$

32.6974

RSL_389

$-34.088055$

22.26111 
RSL_390

$-34.090833$

22.2475

RSL_391

$-34.083833$

22.253611

RSL_392

RSL_393

$-34.064722$

22.208888

RSL_394

$-34.089444$

22.263888

RSL_395

$-34.073055$

22.26222

RSL_396

$-34.068888$

22.257777

RSL_397

$-34.078055$

22.253611

RSL_398

$-34.1025$

22.256388

RSL_399

$-34.056388$

22.239722

RSL_400

$-34.056111$

22.240558

RSL_401

$-34.055833$

22.246388

RSL_402

$-34.055833$

22.246388

RSL_403

$-34.055833$

22.246388

RSL_404

$-34.055833$

22.246388 
RSL_405

$-34.055833$

22.246388

RSL_406

RSL_407

RSL_407

$-34.056111$

22.240555

RSL_408

$-33.152777$

18.038333

RSL_408

$-33.152777$

$-33.152777$

$-33.152777$

18.038333

RSL_408

RSL_412

$-29.98444$

RSL_414

$-31.987624$

RSL_415

$-27.878164$

RSL_417

$-29.998439$

RSL_418

$-29.998439$

RSL_420

$-29.666704$
30.96545

29.152102

32.3299

30.948873

30.948873

18.038333

31.122319 
RSL_421

$-27.969495$

32.384141

RSL_422

$-30.25$

$-30.25$

$-30.25$

30.816667

RSL_423

$-30.25$

30.816667

RSL_444

$-33.87108$

25.623122 


\begin{tabular}{|c|c|c|}
\hline Site & Subsite & Nation \\
\hline $\begin{array}{c}\text { Benguela } \\
\text { Near Site Benguela A } \\
\text { (Walker et al. 2016) }\end{array}$ & $\begin{array}{c}\text { Near Site Benguela A } \\
\text { (Walker et al. 2016) }\end{array}$ & Angola \\
\hline $\begin{array}{c}\text { Benguela } \\
\text { Near Site Benguela A } \\
\text { (Walker et al. 2016) }\end{array}$ & $\begin{array}{c}\text { Near Site Benguela A } \\
\text { (Walker et al. 2016) }\end{array}$ & Angola \\
\hline Mossamedes & & Angola \\
\hline $\begin{array}{c}\text { Benguela } \\
\text { Benguela A }\end{array}$ & Benguela A & Angola \\
\hline $\begin{array}{c}\text { Benguela } \\
\text { Benguela A }\end{array}$ & Benguela A & Angola \\
\hline $\begin{array}{c}\text { Benguela } \\
\text { Benguela A }\end{array}$ & Benguela A & Angola \\
\hline $\begin{array}{c}\text { Benguela } \\
\text { Benguela A }\end{array}$ & Benguela A & Angola \\
\hline $\begin{array}{l}\text { Benguela } \\
\text { Site B }\end{array}$ & Site B & Angola \\
\hline $\begin{array}{l}\text { Benguela } \\
\text { Site B }\end{array}$ & Site B & Angola \\
\hline $\begin{array}{l}\text { Benguela } \\
\text { Site C }\end{array}$ & Site C & Angola \\
\hline $\begin{array}{l}\text { East London } \\
\text { Blind River }\end{array}$ & Blind River & South Africa \\
\hline $\begin{array}{l}\text { East London } \\
\text { Blind River2 }\end{array}$ & Blind River2 & South Africa \\
\hline Dana Bay & & South Africa \\
\hline $\begin{array}{c}\text { Dana Bay } \\
1\end{array}$ & 1 & South Africa \\
\hline
\end{tabular}


Dana Bay

2

South Africa

Swartvlei

Estuary mouth 1

Estuary mouth 1

South Africa

Swartvlei

Swartvlei estuary mouth 2

Swartvlei estuary mouth 2

South Africa

Groot Brak Estuary Mouth

South Africa

Cape Agulhas

South Africa

Swartvlei

Swartvlei Estuary Mouth 3 Swartvlei Estuary Mouth 3

South Africa

Swartvlei

Swartvlei Estuary Mouth 4 Swartvlei Estuary Mouth 4

South Africa

Swartvlei

Swartvlei Estuary mouth 5 Swartvlei Estuary mouth 5

South Africa

Groot Brak Estuary Mouth

Groot Brak Estuary Mouth

Groot Brak Estuary Mouth

South Africa

$\begin{array}{lll}\begin{array}{c}\text { Nahoon Point } \\ \text { Footprints site }\end{array} & \text { Footprints site } & \text { South Africa } \\ \begin{array}{c}\text { Nahoon Point } \\ \text { East Beach1 }\end{array} & \text { East Beach1 } & \text { South Africa }\end{array}$

Nahoon Point

East Beach

East Beach

South Africa

Sodwana Bay

Two Mile Reef

Two Mile Reef

South Africa

Sodwana Bay

Two Mile Reef 2

Two Mile Reef 2

South Africa

Mossel Bay

$-34 m$

$-34 m$

South Africa 
Mossel Bay

$-33 m$

Mossel Bay

$-30 m$

Mossel Bay

$-30 \mathrm{~m} \mathrm{~B}$

Mossel Bay

Shoreline

Mossel Bay

$-35 m$

Mossel Bay

$-26 m$

Mossel Bay

$-17 m$

Mossel Bay

$-25 m$

Mossel Bay

$-38 m$

Groot Brak Estuary Mouth

Site A

Groot Brak Estuary Mouth

Site B

Groot Brak Estuary Mouth

Site C

Site C

South Africa

Groot Brak Estuary Mouth Site C

Groot Brak Estuary Mouth Site C

Site C

South Africa

Groot Brak Estuary Mouth Site C

$-33 m$

South Africa

South Africa

South Africa

South Africa

South Africa

South Africa

South Africa

South Africa

South Africa

South Africa

South Africa

South Africa

Site C

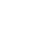




\section{Groot Brak Estuary Mouth} Site C

\section{Groot Brak Estuary Mouth} Site C

\section{Groot Brak Estuary Mouth} Site B.

\section{Groot Brak Estuary Mouth} Site B.

South Africa

\section{Site C}

South Africa

Site B.

South Africa

Site B.

South Africa

\section{Langebaan \\ Hominid footprint site}

Langebaan
Hominid footprint site

Langebaan

Hominid footprint site

Langebaan
Hominid footprint site

Langebaan

Hominid footprint site

Durban Bluff

Reunion Rocks

Coffee Bay

Phinda Game Reserve
South Africa

South Africa

Hominid footprint site

Hominid footprint site

South Africa

Hominid footprint site

South Africa
South Africa

\section{South Africa}

Isipingo

South Africa

Isipingo

South Africa

Umdloti

South Africa 
Aliwal Shoal

South Africa

Aliwal Shoal

South Africa

Aliwal Shoal

South Africa

Aliwal Shoal

South Africa

Swartkops Estuary Mouth Deal Party 


\begin{tabular}{|l|l|l|}
\hline Region & Type of datapoint & RSL Indicator \\
\hline Benguela & Sea Level Indicator & Lagoonal deposit \\
\hline
\end{tabular}

Benguela Sea Level Indicator Lagoonal deposit

$\begin{array}{ll}\text { Namibe } & \text { Seach deposit or } \\ \text { beachrock }\end{array}$

Benguela Sea Level Indicator Lagoonal deposit

Benguela Sea Level Indicator Lagoonal deposit

Benguela Sea Level Indicator Lagoonal deposit

Benguela Sea Level Indicator Lagoonal deposit

Benguela Sea Level Indicator Lagoonal deposit

Benguela Sea Level Indicator Lagoonal deposit

Benguela Sea Level Indicator Lagoonal deposit

Eastern Cape Sea Level Indicator Lagoonal deposit

Eastern Cape Sea Level Indicator Lagoonal deposit Western Cape $\quad$ Sea Level Indicator $\quad$ Shoreface/foreshore

Western Cape

Sea Level Indicator

Shoreface/foreshore contact 


\begin{tabular}{|c|c|c|}
\hline Western Cape & Terrestrial Limiting & $\begin{array}{c}\text { The datapoint is a marine } \\
\text { or terrestrial limiting } \\
\text { indicator }\end{array}$ \\
\hline Western Cape & Sea Level Indicator & $\begin{array}{c}\text { Tidal inlet facies (Western } \\
\text { Cape, South Africa) }\end{array}$ \\
\hline Western Cape & Sea Level Indicator & $\begin{array}{c}\text { Tidal inlet facies (Western } \\
\text { Cape, South Africa) }\end{array}$ \\
\hline Western Cape & Sea Level Indicator & Beach swash deposit \\
\hline Western Cape & Sea Level Indicator & Foreshore deposits \\
\hline Western Cape & Sea Level Indicator & $\begin{array}{c}\text { Tidal inlet facies (Western } \\
\text { Cape, South Africa) }\end{array}$ \\
\hline Western Cape & Terrestrial Limiting & $\begin{array}{c}\text { The datapoint is a marine } \\
\text { or terrestrial limiting } \\
\text { indicator }\end{array}$ \\
\hline Western Cape & Terrestrial Limiting & $\begin{array}{c}\text { The datapoint is a marine } \\
\text { or terrestrial limiting } \\
\text { indicator }\end{array}$ \\
\hline Western Cape & Terrestrial Limiting & $\begin{array}{c}\text { The datapoint is a marine } \\
\text { or terrestrial limiting } \\
\text { indicator }\end{array}$ \\
\hline Eastern Cape & Terrestrial Limiting & $\begin{array}{c}\text { The datapoint is a marine } \\
\text { or terrestrial limiting } \\
\text { indicator }\end{array}$ \\
\hline Eastern Cape & Sea Level Indicator & $\begin{array}{l}\text { Beach deposit or } \\
\text { beachrock }\end{array}$ \\
\hline Eastern Cape & Terrestrial Limiting & $\begin{array}{c}\text { The datapoint is a marine } \\
\text { or terrestrial limiting } \\
\text { indicator }\end{array}$ \\
\hline KwaZulu-Natal & Sea Level Indicator & $\begin{array}{l}\text { Beach deposit or } \\
\text { beachrock }\end{array}$ \\
\hline KwaZulu-Natal & Sea Level Indicator & $\begin{array}{l}\text { Beach deposit or } \\
\text { beachrock }\end{array}$ \\
\hline Western Cape & Terrestrial Limiting & $\begin{array}{c}\text { The datapoint is a marine } \\
\text { or terrestrial limiting } \\
\text { indicator }\end{array}$ \\
\hline
\end{tabular}


Western Cape

Western Cape

Western Cape

Sea Level Indicator

Sea Level Indicator

Terrestrial Limiting

The datapoint is a marine or terrestrial limiting

indicator

Foreshore deposits

The datapoint is a marine or terrestrial limiting indicator

The datapoint is a marine or terrestrial limiting indicator

Foreshore deposits
Western Cape

Sea Level Indicator

Foreshore deposits

Western Cape

Sea Level Indicator

Foreshore deposits

The datapoint is a marine

Western Cape

Terrestrial Limiting

or terrestrial limiting

indicator

Western Cape

Marine Limiting

Foreshore deposits
Western Cape

Western Cape

Terrestrial Limiting

Terrestrial Limiting

Foreshore deposits

Western Cape

Terrestrial Limiting

Foreshore deposits 
Western Cape

Terrestrial Limiting

Marine Limiting

Sea Level Indicator

Western Cape

Western Cape

Sea Level Indicator

Sea Level Indicator

Sea Level Indicator

Sea Level Indicator

Sea Level Indicator

Sea Level Indicator

Western Cape

KwaZulu-Natal

Terrestrial Limiting

Sea Level Indicator

KwaZulu-Natal Sea Level Indicator Shore platform

KwaZulu-Natal Sea Level Indicator Shore platform

Shoreface/foreshore contact

Shoreface/foreshore contact

Lagoonal deposit

Lagoonal deposit

Lagoonal deposit

Lagoonal deposit

Lagoonal deposit

The datapoint is a marine or terrestrial limiting indicator

Shore platform

KwaZulu-Natal

Sea Level Indicator

Beach swash deposit

KwaZulu-Natal

Sea Level Indicator

Shore platform 


\begin{tabular}{|c|c|c|}
\hline KwaZulu-Natal & Marine Limiting & $\begin{array}{c}\text { The datapoint is a marine } \\
\text { or terrestrial limiting } \\
\text { indicator }\end{array}$ \\
\hline KwaZulu-Natal & Terrestrial Limiting & $\begin{array}{c}\text { The datapoint is a marine } \\
\text { or terrestrial limiting } \\
\text { indicator }\end{array}$ \\
\hline KwaZulu-Natal & Terrestrial Limiting & $\begin{array}{c}\text { The datapoint is a marine } \\
\text { or terrestrial limiting } \\
\text { indicator }\end{array}$ \\
\hline KwaZulu-Natal & Terrestrial Limiting & $\begin{array}{c}\text { The datapoint is a marine } \\
\text { or terrestrial limiting } \\
\text { indicator }\end{array}$ \\
\hline KwaZulu-Natal & Terrestrial Limiting & $\begin{array}{c}\text { The datapoint is a marine } \\
\text { or terrestrial limiting } \\
\text { indicator }\end{array}$ \\
\hline Eastern Cape & Sea Level Indicator & Lagoonal deposit \\
\hline
\end{tabular}




\begin{tabular}{|c|c|c|}
\hline RSL indicator description & $\begin{array}{c}\text { Elevation measurement } \\
\text { technique }\end{array}$ & Elevation $(\mathrm{m})$ \\
\hline $\begin{array}{l}\text { The aatea snelis are trom a } \\
\text { sandy deposit interpreted } \\
\text { as lagoonal from the } \\
\text { fainal rnntent }\end{array}$ & Not reported & 11 \\
\hline
\end{tabular}

Shell from a lagoonal assemblage

Not reported

11

Beach deposit on marine terrace

Not reported

15

Not reported

25

Not reported

25

Not reported

25

Not reported

25

Not reported

25

Not reported

25

Not reported

25

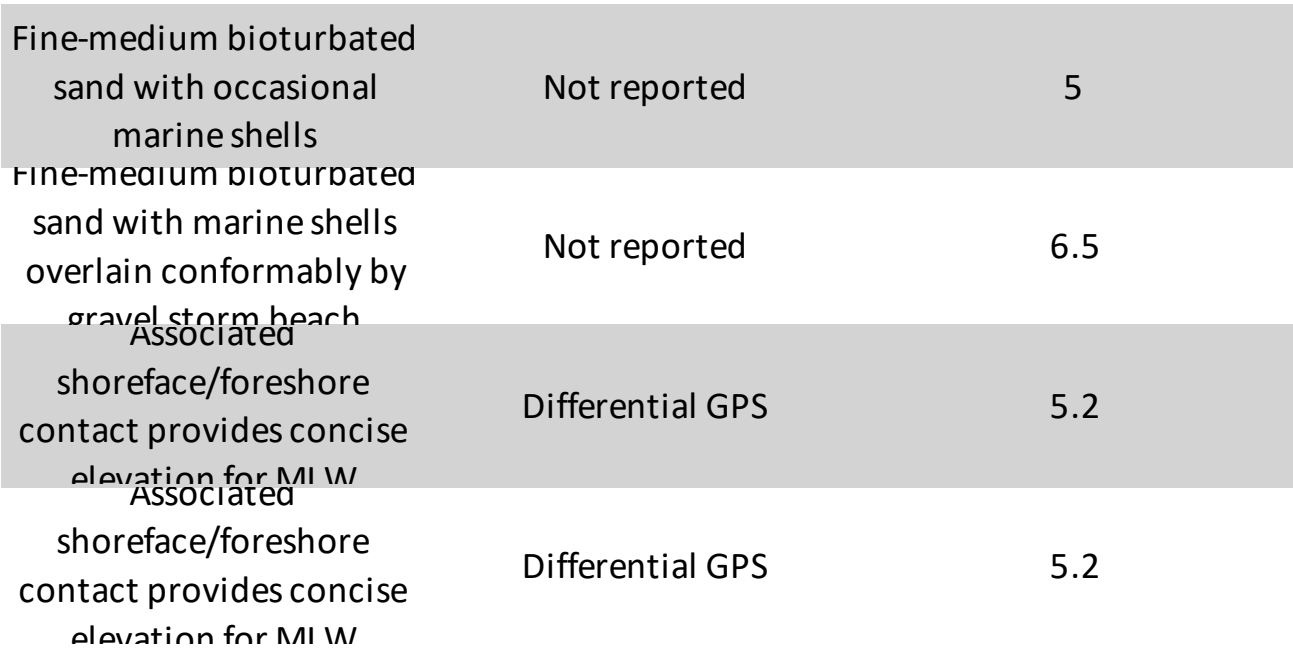


Ilaal ınıet racıes (aatea) overlain by aeolian facies (also dated). Sea level

Total station or determined hy

Sedimentary facies indicative of tidal inlet depositional environment

Auto/hand level

Sandy beach berm on high energy coast

Total station or

Auto/hand level

Total station or

Auto/hand level

\section{Sandy foreshore deposits overlying gravel foreshore \\ Lower part of tidal inlet unit \\ Aeolianite unit overlying tidal inlet facies apparently conformably}

Total station or

Auto/hand level

Total station or

Auto/hand level

Total station or

Auto/hand level

Total station or

Auto/hand level

\begin{tabular}{|c|c|c|}
\hline $\begin{array}{l}\text { Aeolianite overlying beach } \\
\text { berm facies. }\end{array}$ & $\begin{array}{l}\text { Total station or } \\
\text { Auto/hand level }\end{array}$ & 10.5 \\
\hline $\begin{array}{l}\text { rootprınt-Dearıng } \\
\text { aeolianite pre-dating } \\
\text { (underlying) beach } \\
\text { denncit of NHN1 }\end{array}$ & Not reported & 4.5 \\
\hline $\begin{array}{l}\text { Coarse grained cemented } \\
\text { shelly beach facies }\end{array}$ & Not reported & 4.25 \\
\hline $\begin{array}{c}\text { Aeolianıte overıyıng } \\
\text { earlier beach facies and } \\
\text { incised by later beach } \\
\text { fariec }\end{array}$ & Not reported & 6 \\
\hline $\begin{array}{l}\text { Submerged beachrock } \\
\text { from continental shelf }\end{array}$ & $\begin{array}{l}\text { Cross-section from } \\
\text { publication }\end{array}$ & -17 \\
\hline $\begin{array}{c}\text { Beachrock on continental } \\
\text { shelf }\end{array}$ & $\begin{array}{c}\text { Cross-section from } \\
\text { publication }\end{array}$ & -44 \\
\hline Aeolianite & Not reported & -34 \\
\hline
\end{tabular}


Aeolianite

Aeolianite

Not reported

Aeolianite

Not reported

$-30$

IVIassive aeposic or

medium- to coarse sand, moderately

Total station or

Auto/hand level

0

Aeolianite

Not reported

IVIassive aeposit or

medium- to coarse sand, moderately

Not reported

Upper shoreface- trough

cross-bedded sands

Not reported

Deposit of beach or

surfzone

Not reported

Aeolianite

Not reported

\begin{tabular}{ccc} 
Upper shoreface unit & $\begin{array}{c}\text { Total station or } \\
\text { Auto/hand level }\end{array}$ & 3.5 \\
\hline Aeolianite & $\begin{array}{c}\text { Total station or } \\
\text { Auto/hand level }\end{array}$ & 11.1 \\
Aeolianite & $\begin{array}{c}\text { Total station or } \\
\text { Auto/hand level }\end{array}$ & 23.5 \\
\hline Aeolianite & $\begin{array}{l}\text { Total station or } \\
\text { Auto/hand level }\end{array}$ & 20.2 \\
\hline Aeolianite & Total station or \\
& Auto/hand level & 13.3 \\
\hline Aeolianite & Total station or \\
& Auto/hand level & 10.4
\end{tabular}




\begin{tabular}{|c|c|c|}
\hline Aeolianite & $\begin{array}{l}\text { Total station or } \\
\text { Auto/hand level }\end{array}$ & 9.5 \\
\hline Upper shoreface & $\begin{array}{l}\text { Total station or } \\
\text { Auto/hand level }\end{array}$ & 6 \\
\hline Beach-surf zone facies & $\begin{array}{l}\text { Total station or } \\
\text { Auto/hand level }\end{array}$ & 9 \\
\hline Beach-surf zone facies & $\begin{array}{l}\text { Total station or } \\
\text { Auto/hand level }\end{array}$ & 9 \\
\hline $\begin{array}{l}\text { Marginal lagoonal deposit } \\
\text { with contemporaneous } \\
\text { aeolian deposits adjacent }\end{array}$ & $\begin{array}{l}\text { Total station or } \\
\text { Auto/hand level }\end{array}$ & 1.3 \\
\hline $\begin{array}{c}\text { Marginal lagoonal deposit } \\
\text { with contemporaneous } \\
\text { aeolian deposits adjacent }\end{array}$ & $\begin{array}{l}\text { Total station or } \\
\text { Auto/hand level }\end{array}$ & 1.3 \\
\hline $\begin{array}{c}\text { Marginal lagoonal deposit } \\
\text { with contemporaneous } \\
\text { aeolian deposits adjacent }\end{array}$ & $\begin{array}{l}\text { Total station or } \\
\text { Auto/hand level }\end{array}$ & 1.3 \\
\hline $\begin{array}{c}\text { Marginal lagoonal deposit } \\
\text { with contemporaneous } \\
\text { aeolian deposits adjacent }\end{array}$ & $\begin{array}{l}\text { Total station or } \\
\text { Auto/hand level }\end{array}$ & 1.3 \\
\hline $\begin{array}{c}\text { Marginal lagoonal deposit } \\
\text { with contemporaneous } \\
\text { aeolian deposits adjacent }\end{array}$ & $\begin{array}{l}\text { Total station or } \\
\text { Auto/hand level }\end{array}$ & 1.3 \\
\hline $\begin{array}{l}\text { tIepnant tusk in eroaed } \\
\text { pothole on wave-cut } \\
\text { surface ca } 6 \text { m ASL. Tusk } \\
\text { miıctnnct-dato formatinn } \\
\text { Iransportéa oyster onn }\end{array}$ & Not reported & 6 \\
\hline $\begin{array}{c}\text { shore platform in case at } \\
4.5 \mathrm{~m} \text {. regarded as } \\
\text { rnntomnnranenuchy }\end{array}$ & Not reported & 4.5 \\
\hline 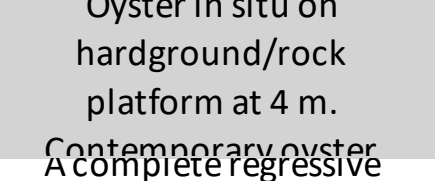 & Not reported & 4 \\
\hline $\begin{array}{l}\text { beach facies assemblage } \\
\text { from subtidal through }\end{array}$ & $\begin{array}{l}\text { Total station or } \\
\text { Auto/hand level }\end{array}$ & 6 \\
\hline $\begin{array}{l}\text { into earlier (also } \\
\text { presumed } 5 \text { e) regressive }\end{array}$ & $\begin{array}{l}\text { Total station or } \\
\text { Auto/hand level }\end{array}$ & 5 \\
\hline $\begin{array}{c}\text { snore platiorm at } 4 \mathrm{~m} \\
\text { above adjacent } \\
\text { contemporary shore } \\
\text { nlatfnrm }\end{array}$ & Differential GPS & 4 \\
\hline
\end{tabular}


Corals growing on

erosional terrace

Aeolianite

Differential GPS

Total station or

Auto/hand level

4

Aeolianite

Differential GPS

$-15$

Aeolianite

Differential GPS

$-16$

Aeolianite

Differential GPS

$-16$

snelIs trom terrace at +0. .

$\mathrm{m}$, deemed contemporary with a $+8 m$ terrace

Not reported

8

inctraam 


\begin{tabular}{|c|c|c|}
\hline Elevation error $(\mathrm{m})$ & $\begin{array}{c}\text { Paleo water depth } \\
\text { estimate }(\mathrm{m})\end{array}$ & $\begin{array}{c}\text { Upper limit of living range } \\
(\mathrm{m})\end{array}$ \\
\hline 1
\end{tabular}

1

1

1

1

1

1

1

1

1

0.5

0.5

0.02

0.02 
0.02

2

0.1

1

0.1

0.1

0.1

0.1

0.1

0.1

1.75

0.1

2

2

1 
1

1

1

0.1

1

1

1

1

1

0.05

0.05

0.05

0.05

0.05

0.05 
0.05

0.05

0.05

0.05

0.1

0.1

0.1

0.1

0.1

1

1

0.1

1

1

0.1 
0.1

1

1

1

1

1 


\begin{tabular}{|c|c|c|}
\hline $\begin{array}{l}\text { Lower limit of living range } \\
\text { (m) }\end{array}$ & RWL & IR \\
\hline & -0.5 & 3 \\
\hline & -0.5 & 3 \\
\hline & 0 & 4 \\
\hline & -0.5 & 3 \\
\hline & -0.5 & 3 \\
\hline & -0.5 & 3 \\
\hline & -0.5 & 3 \\
\hline & -0.5 & 3 \\
\hline & -0.5 & 3 \\
\hline & -0.5 & 3 \\
\hline & -1 & 4 \\
\hline & -1 & 4 \\
\hline & -1 & 0 \\
\hline & -1 & 0 \\
\hline
\end{tabular}


$-1$

$-2$

$-2$

1.75

0

$-1.5$

0

1

0

1

0

1 
$-1$

$-1$

$-11.5$

$-1$ 


$\begin{array}{ll}-1 & 4 \\ -1 & 4 \\ 0 & 2 \\ 0 & 2 \\ 0 & 2 \\ 0 & \\ 0 & 2 \\ 0 & 2\end{array}$

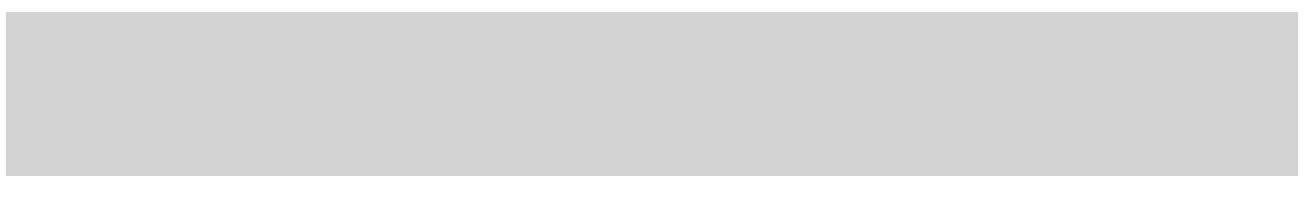

$2.5 \quad 5$

$1 \quad 0$

$\begin{array}{ll}1.5 & 1\end{array}$

$\begin{array}{ll}0 & 1 \\ 0 & 0\end{array}$




\begin{tabular}{|c|c|c|}
\hline Vertical datum & Paleo RSL (m) & Paleo RSL uncertainty (m) \\
\hline $\begin{array}{c}\text { Mean Sea Level / General } \\
\text { definition }\end{array}$ & 11.5 & 1.8 \\
\hline $\begin{array}{c}\text { Mean Sea Level / General } \\
\text { definition }\end{array}$ & 11.5 & 1.8 \\
\hline $\begin{array}{c}\text { Mean Sea Level / General } \\
\text { definition }\end{array}$ & 15 & 2.23 \\
\hline $\begin{array}{c}\text { Mean Sea Level / General } \\
\text { definition }\end{array}$ & 25.5 & 1.8 \\
\hline $\begin{array}{c}\text { Mean Sea Level / General } \\
\text { definition }\end{array}$ & 25.5 & 1.8 \\
\hline $\begin{array}{c}\text { Mean Sea Level / General } \\
\text { definition }\end{array}$ & 25.5 & 1.8 \\
\hline $\begin{array}{c}\text { Mean Sea Level / General } \\
\text { definition }\end{array}$ & 25.5 & 1.8 \\
\hline $\begin{array}{c}\text { Mean Sea Level / General } \\
\text { definition }\end{array}$ & 25.5 & 1.8 \\
\hline $\begin{array}{c}\text { Mean Sea Level / General } \\
\text { definition }\end{array}$ & 25.5 & 1.8 \\
\hline $\begin{array}{c}\text { Mean Sea Level / General } \\
\text { definition }\end{array}$ & 25.5 & 1.8 \\
\hline $\begin{array}{c}\text { Mean Sea Level / General } \\
\text { definition }\end{array}$ & 6 & 2.06 \\
\hline $\begin{array}{c}\text { Mean Sea Level / General } \\
\text { definition }\end{array}$ & 7.5 & 2.06 \\
\hline Mean Low Tide (MLT) & 6.2 & 0.02 \\
\hline Mean Low Tide (MLT) & 6.2 & 0.02 \\
\hline
\end{tabular}


Mean Sea Level / General definition

Mean Sea Level / General definition

Mean Sea Level / General definition

\section{Mean Sea Level / General} definition

Mean Sea Level / General definition

\section{Mean Sea Level / General definition}

Mean Sea Level / General definition

\section{Mean Sea Level / General} definition

\section{Mean Sea Level / General} definition

\section{Mean Sea Level / General definition}

Mean Sea Level / General definition

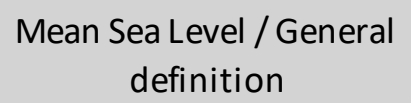

Mean Sea Level / General definition

\section{Mean Sea Level / General} definition 
Mean Sea Level / General definition

\section{Mean Sea Level / General definition}

Mean Sea Level / General definition

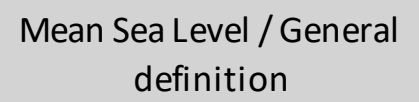

Mean Sea Level / General definition

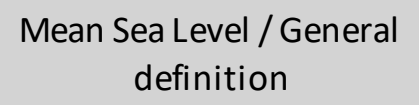

Mean Sea Level / General definition

Mean Sea Level / General definition

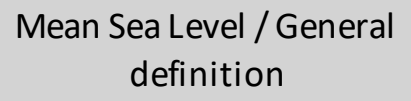

Mean Sea Level / General definition 


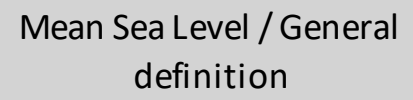

Mean Sea Level / General definition

\begin{tabular}{|c|c|c|}
\hline $\begin{array}{c}\text { Mean Sea Level / General } \\
\text { definition }\end{array}$ & 10 & 2 \\
\hline $\begin{array}{c}\text { Mean Sea Level / General } \\
\text { definition }\end{array}$ & 10 & 2 \\
\hline $\begin{array}{c}\text { Mean Sea Level / General } \\
\text { definition }\end{array}$ & 1.3 & 1 \\
\hline $\begin{array}{c}\text { Mean Sea Level / General } \\
\text { definition }\end{array}$ & 1.3 & 1 \\
\hline $\begin{array}{c}\text { Mean Sea Level / General } \\
\text { definition }\end{array}$ & 1.3 & 1 \\
\hline $\begin{array}{c}\text { Mean Sea Level / General } \\
\text { definition }\end{array}$ & 1.3 & 1 \\
\hline $\begin{array}{c}\text { Mean Sea Level / General } \\
\text { definition }\end{array}$ & 1.3 & 1 \\
\hline $\begin{array}{c}\text { Mean Sea Level / General } \\
\text { definition }\end{array}$ & & \\
\hline $\begin{array}{c}\text { Mean Sea Level / General } \\
\text { definition }\end{array}$ & 2 & 2.69 \\
\hline $\begin{array}{c}\text { Mean Sea Level / General } \\
\text { definition }\end{array}$ & 3 & 0.1 \\
\hline $\begin{array}{c}\text { Mean Sea Level / General } \\
\text { definition }\end{array}$ & 4.5 & 1.11 \\
\hline $\begin{array}{c}\text { Mean Sea Level / General } \\
\text { definition }\end{array}$ & 5 & 1.11 \\
\hline $\begin{array}{c}\text { Mean Sea Level / General } \\
\text { definition }\end{array}$ & 4 & 0.1 \\
\hline
\end{tabular}


Mean Sea Level / General definition

Mean Sea Level / General definition

Mean Sea Level / General definition

Mean Sea Level / General definition

Mean Sea Level / General definition

Mean Sea Level / General definition 


\begin{tabular}{|c|c|c|}
\hline Dating technique & Timing constraint & Originally reported ID \\
U-Series & Equal to & AN57-1
\end{tabular}

$\begin{array}{lll}\text { U-Series } & \text { Equal to } & \text { AN57-2 }\end{array}$

$\begin{array}{lll}\text { U-Series } & \text { Equal to } & \text { AN40-2 }\end{array}$

Luminescence Equal to BNG10-6

$\begin{array}{lll}\text { Luminescence } & \text { Equal to } & \text { BNG10-7 }\end{array}$

$\begin{array}{lll}\text { Luminescence } & \text { Equal to }\end{array}$

$\begin{array}{lll}\text { Luminescence } & \text { Equal to } & \text { BNG10-9 }\end{array}$

$\begin{array}{lll}\text { Luminescence } & \text { Equal to }\end{array}$

$\begin{array}{lll}\text { Luminescence } & \text { Equal to } & \text { BNG10-11 }\end{array}$

$\begin{array}{lll}\text { Luminescence } & \text { Equal to } & \text { BNG10-12 }\end{array}$

Luminescence Equal to Sample1

Luminescence $\quad$ Equal to $\quad$ Sample2

$\begin{array}{lll}\text { Luminescence } & \text { Equal to } & \text { Dana7 }\end{array}$

Luminescence Equal to Dana6 
Luminescence

Equal to

UoW-234

Luminescence

Equal to

SHFD07072

Luminescence

Equal to

UOW-232

Luminescence

Equal to

SHFD05019

Luminescence

Equal to

SHFD04288

Luminescence

Equal to

SHFD05038

Luminescence

Equal to

UOW-235

Luminescence

Equal to

UOW-233

Luminescence

Equal to

NN1

Luminescence

Equal to

NHN1

Luminescence

Equal to

NHN2

U-Series

Equal to

PTA-U-435

U-Series

Equal to

PTA-U-487

Luminescence

Equal to

HC30 
Luminescence

Luminescence

Equal to

HC35

Luminescence

Equal to

HC36

Luminescence

Equal to

$\mathrm{HCSH}$

Luminescence

Equal to

HC29

Luminescence

Equal to

HC40

Luminescence

Equal to

HC32

Luminescence

Equal to

HC39

Luminescence

Equal to

HC24

Luminescence

Equal to

142833

Luminescence

Equal to

142832

Luminescence

Equal to

157206

Luminescence

Equal to

157205

Luminescence

Equal to

157204 
Luminescence

Equal to

142829

Luminescence

Equal to

142831

Luminescence

Equal to

142830

U-Series

Equal to

RB97-04

U-Series

Equal to

RB97-05

Luminescence

Equal to

RB97-01

Luminescence

Equal to

RB97-02

Luminescence

Equal to

RB97-03

U-Series

Older than

U415

U-Series

Equal to

PTA-U568

U-Series

Equal to

PTA-U565

Stratigraphic constraint

Younger than

Isipingo Formation on

Durban Bluff

Stratigraphic constraint

Younger than

Isipingo Formation on Durban Bluff

Stratigraphic constraint

Younger than

Isipingo Formation on

Durban Bluff 
U-Series

U-Series

Luminescence

U-Series

Luminescence

AAR

Equal to
PTA-U565

GC-4

GC-4

GC-7

GC-7

PE-557 


\begin{tabular}{|c|c|c|}
\hline Analysis ID & Material_type & Reported age (ka) \\
\hline GI84-001-001 & Mollusk or algae & \\
\hline GI84-00-001 & Mollusk or algae & \\
\hline GI84-003-001 & Mollusk or algae & \\
\hline WA16-001-001 & OSL & 57.8 \\
\hline WA16-002-001 & OSL & 36.8 \\
\hline WA16-003-001 & OSL & 47 \\
\hline WA16-004-001 & OSL & 53.9 \\
\hline WA16-005-001 & OSL & 42.5 \\
\hline WA16-006-001 & OSL & 46.8 \\
\hline WA16-007-001 & OSL & 44.7 \\
\hline WA08-001-001 & OSL & 119 \\
\hline 1 & OSL & 118 \\
\hline R012-001-001 & OSL & 125 \\
\hline R012-002-001 & OSL & 116 \\
\hline
\end{tabular}


R012-003-001

OSL

CA10-001-001

OSL

127

CA10-002-001

OSL

130

CA10-003-001

OSL

125

CA10-004-001

OSL

118

CA10-005-001

OSL

138

CA10-006-001

OSL

112

CA10-007-001

OSL

113

CA10-008-001

OSL

122

JA09-001-001

OSL

124.8

JA09-002-001

OSL

117.3

JA09-003-001

OSL

115.8

RA94-001-001

Mollusk or algae

RA94-002-001

Mollusk or algae

CA14-004-001

OSL

142 
CA14-001-001

CA14-005-001

CA14-002-001

CA14-003-001

CA14-006-001

CA14-007-001

CA14-008-001

CA14-009-001

CA14-010-001

CA18-007-001

CA18-001-001

CA18-002-001

CA18-008-001

CA18-009-001

CA18-010-001
OSL

OSL

134

OSL

OSL

122

OSL

122

OSL

117

OSL

OSL

103

OSL

87

OSL

116.1

OSL

119.7

OSL

118

OSL

123.4

OSL

135.4

OSL

128.4 
CA18-011-001

CA18-006-001

CA18-003-001

CA18-004-001

R097-001-001

R097-002-001

R097-001-001

R097-002-001

R097-003-001

RA93-001-001

RA02-001-001

RA02-002-001
OSL

128.1

OSL

111.2

OSL

124.3

OSL

116.3

Mollusk or algae

Mollusk or algae

IRSL

TL

228

IRSL

107

Mollusk or algae

Mollusk or algae

Mollusk or algae 
RA02-002-001

B012-001-001

B012-001-001

B012-002-001

B012-002-001

DA80-001-001
Mollusk or algae

Mollusk or algae

OSL

127

Mollusk or algae

OSL

93

Estuarine mollusc 


\begin{tabular}{|l|c|c|}
\hline Reported age uncertainty $(\mathrm{ka})$ & $\begin{array}{c}\text { U-Series recalculated age } \\
(\mathrm{ka})\end{array}$ & $\begin{array}{c}\text { U-Series recalculate age } \\
\text { uncertainty }(\mathrm{ka})\end{array}$ \\
\hline
\end{tabular}

11.2

3.4

5.9

6.2

3.7

7.9

5.6

9

7

9 
9

5.7

8.2

6.7

7.2

7.3

5.1

5.6

6.9

5.2

6.2

7.5 
1.4

13

12

15

10

11

9

8

9

7.3

8.9

8.4

6.9

7.9

9.2 
8.4

7.5

8.6

9.2

18

25

7 


\begin{tabular}{|c|c|c|}
\hline $\begin{array}{c}\text { U-Series corrected age } \\
\text { (speleothems, ka) }\end{array}$ & $\begin{array}{c}\text { U-Series corrected age } \\
\text { uncertainty } \\
\text { (speleothems, ka) }\end{array}$ & $\begin{array}{c}\text { Stratigraphy Upper Age } \\
\text { (ka) }\end{array}$ \\
0 \\
0
\end{tabular}

130

130

57

57

57 
130

130

130

130

130

130

130

130

130

130

130

130

130 


\begin{tabular}{|c|c|c|}
\hline $\begin{array}{c}\text { Stratigraphy Lower Age } \\
\text { (ka) }\end{array}$ & MIS limit & Marine Isotopic Stage \\
\hline 71 & Equal to & MIS 5 \\
\hline 71 & Equal to & MIS 5 \\
\hline 71 & Equal to & MIS 5 \\
\hline 29 & Equal to & MIS 3 \\
\hline 29 & Equal to & MIS 3 \\
\hline 29 & Equal to & MIS 3 \\
\hline & Equal to & \\
\hline & Equal to & \\
\hline & Equal to & \\
\hline
\end{tabular}

Equal to

Equal to

Equal to

Equal to

Equal to 
Equal to

71

Equal to

MIS 5

71

Equal to

MIS 5

71

Equal to

MIS 5

71

Equal to

MIS 5

71

Equal to

MIS 5

71

Equal to

MIS 5

71

Equal to

MIS 5

71

Equal to

MIS 5

71

Equal to

MIS 5

71

Equal to

MIS 5

71

Equal to

MIS 5

71

Equal to

MIS 5

71

Equal to

MIS 5

Equal to 
Equal to

Equal to

Equal to

Equal to

Equal to

Equal to

Equal to

Equal to

Equal to

Equal to

Equal to

Equal to

71

Equal to

MIS 5

71

Equal to

MIS 5

71

Equal to

MIS 5 
Equal to

Equal to

Equal to

71

Equal to

MIS 5

71

Equal to

MIS 5

Equal to

Equal to

Equal to

71

Equal to

MIS 5

71

Equal to

MIS 5

71

Equal to

MIS 5

191

Equal to

MIS 7

191

Equal to

MIS 7

191

Equal to

MIS 7 
Equal to

Equal to 


\begin{tabular}{|c|c|c|}
\hline $\begin{array}{l}\text { Quality of RSL } \\
\text { information }\end{array}$ & $\begin{array}{l}\text { Quality of age } \\
\text { information }\end{array}$ & Reference(s) \\
\hline 2 & 1 & Giresse et al., 1984 \\
\hline 1 & 2 & Giresse et al., 1984 \\
\hline 1 & 1 & Giresse et al., 1984 \\
\hline 3 & 4 & Walker et al., 2016 \\
\hline 3 & 4 & Walker et al., 2016 \\
\hline 3 & 4 & Walker et al., 2016 \\
\hline 3 & 4 & Walker et al., 2016 \\
\hline 3 & 4 & Walker et al., 2016 \\
\hline 3 & 4 & Walker et al., 2016 \\
\hline
\end{tabular}

3

4

Walker et al., 2016

3

4

Wang et al., 2008

3

4

Wang et al., 2008

0

0

Roberts, Karkanas et al., 2012

0

Roberts, Karkanas et al., 2012 
0

0

5

4

3

3

4

1

1

1

1

3

1

4

3

1
Roberts, Karkanas et al., 2012

Carr et al., 2010

Carr et al., 2010

Carr et al., 2010

Carr et al., 2010

Carr et al., 2010

Carr et al., 2010

Carr et al., 2010

Carr et al., 2010

Jacobs and Roberts, 2009

Jacobs and Roberts, 2009

Jacobs and Roberts, 2009

Ramsay, 1994

Ramsay, 1994

Cawthra 2014 
1

1

1

3

1

3

2

3

1

1

1

1

1

1

1
4

4

Cawthra 2014

Cawthra 2014

Cawthra 2014

Cawthra 2014

Cawthra 2014

Cawthra 2014

Cawthra 2014

Cawthra 2014

Cawthra et al., 2018

Cawthra et al., 2018

Cawthra et al., 2018

Cawthra et al., 2018

Cawthra et al., 2018

Cawthra et al., 2018 
Cawthra et al., 2018

Roberts and Berger 1997

3 MR Cooper, 1999

4 1

Cooper and Flores, 1991

Porat and Botha, 2008

4

Cooper and Flores, 1991

Porat and Botha, 2008 
Hobday, 1975

1

Ramsay, 1994

Ramsay and Cooper, 2002

1

4

Bosman, 2012

1

4

Bosman, 2012

1

4

Bosman, 2012

1

4

Bosman, 2012

2

2

Davies, 1980 


\begin{tabular}{|l|l|}
\hline Record Created by & Last Update \\
\hline Andrew Cooper & \\
\hline
\end{tabular}

Andrew Cooper

2020-04-08 05:16:22

Andrew Cooper

2020-04-08 05:16:28

Andrew Cooper

2020-04-08 05:16:37

Andrew Cooper

2020-04-08 05:16:37

Andrew Cooper

2020-04-08 05:16:37

Andrew Cooper

2020-04-08 05:16:37

Andrew Cooper

2020-04-08 05:16:45

Andrew Cooper

2020-04-08 05:16:45

Andrew Cooper

2020-04-08 05:17:38

Andrew Cooper

2020-12-02 14:51:41

Andrew Cooper

2020-12-02 14:52:00

Andrew Cooper

2021-01-21 15:58:14

Andrew Cooper

2021-01-21 15:58:30 
Andrew Cooper

2020-12-16 13:03:46

Andrew Cooper

2020-04-08 05:13:53

Andrew Cooper

2020-04-08 05:14:10

Andrew Cooper

2020-04-08 05:14:27

Andrew Cooper

2020-04-08 05:14:46

Andrew Cooper

2020-04-08 05:15:00

Andrew Cooper

2020-04-08 05:15:08

Andrew Cooper

2020-04-08 05:15:16

Andrew Cooper

2020-04-08 05:15:24

Andrew Cooper

2020-04-08 05:15:32

Andrew Cooper

2020-11-26 14:02:33

Andrew Cooper

2020-04-08 05:15:50

Andrew Cooper

2020-04-08 05:15:58

Andrew Cooper

2020-04-08 05:16:07

Andrew Cooper

2020-04-08 05:17:47 
Andrew Cooper

2020-04-08 05:17:53

Andrew Cooper

2020-04-08 05:17:59

Andrew Cooper

2020-04-08 05:18:07

Andrew Cooper

2020-04-08 05:18:13

Andrew Cooper

2020-04-08 05:18:19

Andrew Cooper

2020-04-08 05:18:26

Andrew Cooper

2020-04-08 05:18:31

Andrew Cooper

2020-04-08 05:18:38

Andrew Cooper

2020-04-08 05:18:46

Andrew Cooper

2020-04-06 10:24:09

Andrew Cooper

2020-04-08 05:18:59

Andrew Cooper

2020-04-08 05:19:10

Andrew Cooper

2020-04-08 05:19:17

Andrew Cooper

2020-04-08 05:19:27

Andrew Cooper

2020-04-07 16:58:53 
Andrew Cooper

2020-04-07 16:59:07

Andrew Cooper

2020-04-07 16:59:19

Andrew Cooper

2021-01-21 15:57:41

Andrew Cooper

2021-01-21 15:57:41

Andrew Cooper

2020-04-07 16:59:45

Andrew Cooper

2020-04-07 16:59:45

Andrew Cooper

2020-04-07 16:59:45

Andrew Cooper

2020-04-07 16:59:45

Andrew Cooper

2020-04-07 16:59:45

Andrew Cooper

2020-04-08 06:17:56

Andrew Cooper

2020-04-07 09:47:55

Andrew Cooper

2020-04-08 10:53:41

Andrew Cooper

2020-04-08 07:38:23

Andrew Cooper

2020-04-08 07:37:53

Andrew Cooper

2020-04-08 09:40:22 
Andrew Cooper

2020-04-08 09:38:41

Andrew Cooper

2020-04-09 05:11:48

Andrew Cooper

2020-04-09 05:11:48

Andrew Cooper

2020-04-09 05:11:17

Andrew Cooper

2020-04-09 05:11:17

Andrew Cooper

2020-12-16 10:26:03 


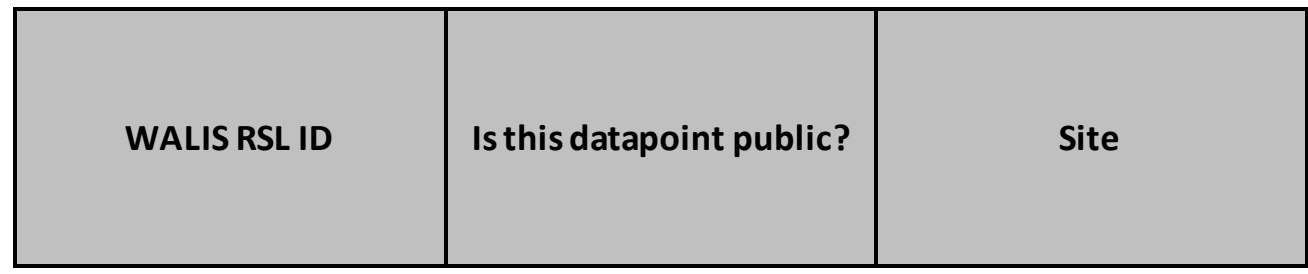

370

1

Benguela

371

1

Benguela

373

1

Benguela

377

1

Benguela

379

1

Benguela

372

1

Mossamedes

365

1

Nahoon Point

366

1

Nahoon Point

367

1

Nahoon Point

414

1

Coffee Bay

444

1

Swartkops Estuary Mouth

3435

0

East London

3436

0

East London

368

1

Sodwana Bay 
Isipingo

Isipingo

Umdloti 


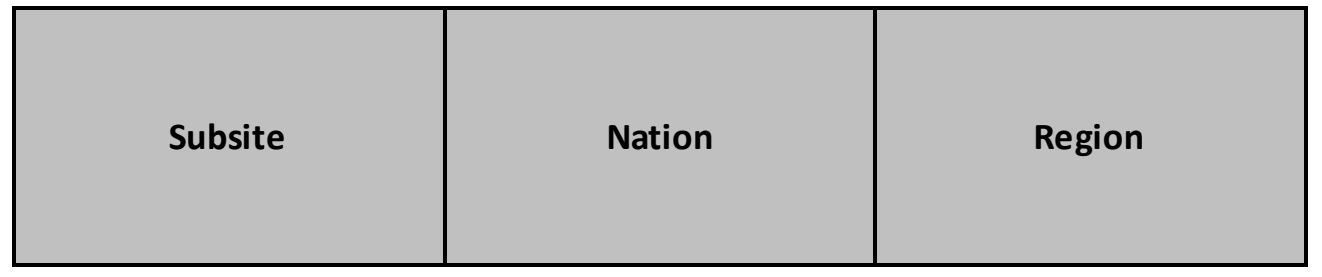

Near Site Benguela A

(Walker et al. 2016)

Angola

Benguela

Near Site Benguela A

(Walker et al. 2016)

Angola

Benguela

Benguela A

Angola

Benguela

Site B

Angola

Benguela

Site C

Angola

Benguela

Angola

Namibe

Footprints site

South Africa

Eastern Cape

East Beach1

South Africa

Eastern Cape

East Beach

South Africa

Eastern Cape

South Africa

Eastern Cape

Deal Party

South Africa

Eastern Cape

Blind River

South Africa

Eastern Cape

Blind River2

South Africa

Eastern Cape

Two Mile Reef

South Africa

KwaZulu-Natal 
Two Mile Reef 2

Reunion Rocks

South Africa

South Africa

South Africa

South Africa

South Africa

South Africa

South Africa

Estuary mouth 1

Swartvlei estuary mouth 2

South Africa

Swartvlei Estuary Mouth 3

Swartvlei Estuary Mouth 4
South Africa

South Africa

South Africa

South Africa

South Africa
KwaZulu-Natal

KwaZulu-Natal

KwaZulu-Natal

KwaZulu-Natal

KwaZulu-Natal

KwaZulu-Natal

KwaZulu-Natal

KwaZulu-Natal

KwaZulu-Natal

Western Cape

Western Cape

Western Cape

Western Cape

Western Cape

Western Cape 
Swartvlei Estuary mouth 5

Groot Brak Estuary Mouth

$-34 m$

$-33 m$

$-30 m$

$-30 m$ B

Shoreline

$-35 m$

$-26 m$

$-17 m$

$-25 m$

$-38 m$

Site A

Site B

Site C
South Africa

South Africa

South Africa

South Africa

South Africa

South Africa

South Africa

South Africa

South Africa

South Africa

South Africa

South Africa

South Africa

South Africa

South Africa
Western Cape

Western Cape

Western Cape

Western Cape

Western Cape

Western Cape

Western Cape

Western Cape

Western Cape

Western Cape

Western Cape

Western Cape

Western Cape

Western Cape

Western Cape 
Site C

Site C

Site C

Site C

Site C

Site B.

Hominid footprint site

1

2
South Africa

South Africa

South Africa

South Africa

South Africa

South Africa

South Africa

South Africa

South Africa

South Africa
Western Cape

Western Cape

Western Cape

Western Cape

Western Cape

Western Cape

Western Cape

Western Cape

Western Cape

Western Cape 


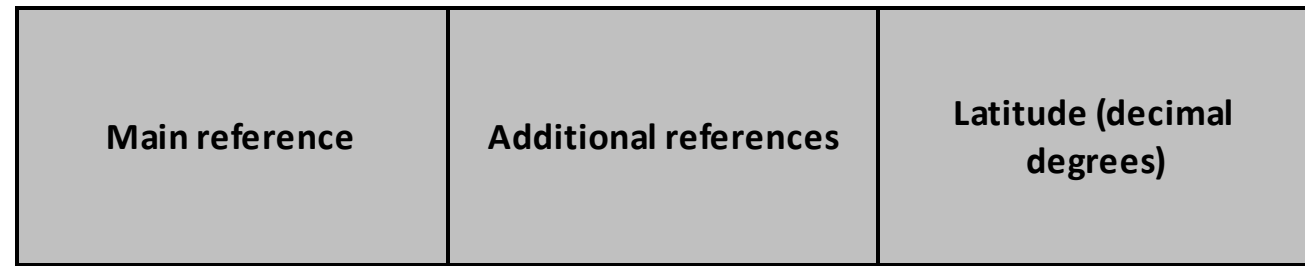

Giresse et al., 1984

N/A

$-12.620277$

Giresse et al., 1984

N/A

$-12.620277$

Walker et al., 2016

N/A

$-12.61$

Walker et al., 2016

N/A

$-12.550833$

Walker et al., 2016

N/A

$-12.544166$

Giresse et al., 1984

N/A

$-15.133055$

Jacobs and Roberts, 2009

N/A

$-32.994722$

Jacobs and Roberts, 2009

N/A

$-33.006027$

Jacobs and Roberts, 2009

N/A

$-33.0225$

Ramsay and Cooper, 2002

N/A

$-31.987624$

Davies, 1980

N/A

$-33.87108$

Wang et al., 2008

N/A

$-33.006137$

Wang et al., 2008

N/A

$-33.006137$

Ramsay, 1994

N/A

$-27.50777$ 
Carr et al., 2010

Carr et al., 2010

Cawthra 2014

Cawthra 2014

Cawthra 2014

Cawthra 2014

Cawthra 2014

Cawthra 2014

Cawthra 2014

Cawthra 2014

Cawthra 2014

Cawthra 2014

Cawthra et al., 2018

Cawthra et al., 2018

Cawthra et al., 2018
N/A

$-34.0325$

N/A

$-34.056111$

N/A

$-34.088055$

N/A

$-34.090833$

N/A

$-34.083833$

N/A

$-34.081666$

N/A

$-34.064722$

N/A

$-34.089444$

N/A

$-34.073055$

N/A

$-34.068888$

N/A

$-34.078055$

N/A

$-34.1025$

N/A

$-34.056388$

N/A

$-34.056111$

N/A

$-34.055833$ 
Cawthra et al., 2018

Cawthra et al., 2018

Cawthra et al., 2018

Cawthra et al., 2018

Cawthra et al., 2018

Cawthra et al., 2018

Roberts and Berger 1997

Roberts, Karkanas et al., 2012

Roberts, Karkanas et al., 2012

Roberts, Karkanas et al., 2012
N/A

$-34.055833$

N/A

$-34.055833$

N/A

$-34.055833$

N/A

$-34.055833$

N/A

$-34.055833$

N/A

$-34.056111$

N/A

$-33.152777$

N/A

$-34.205183$

N/A

$-34.205183$

N/A

$-34.205183$ 


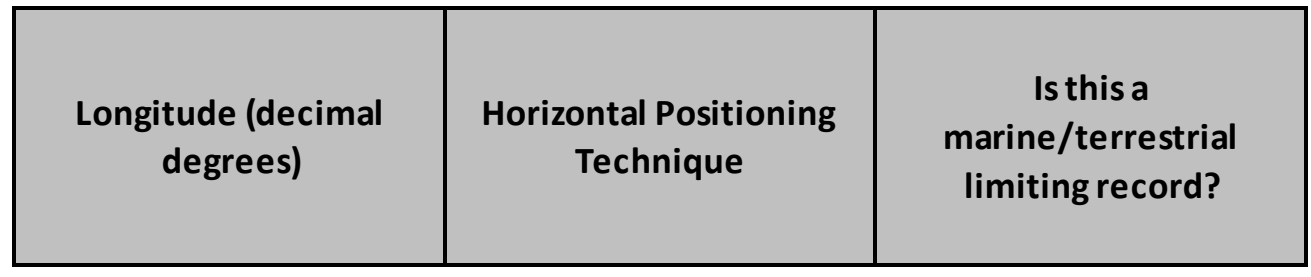

13.243611

Not Specified

Sea Level Indicator

13.243611

Not Specified

Sea Level Indicator

13.365

Not Specified

Sea Level Indicator

13.439722

Not Specified

Sea Level Indicator

13.463888

Not Specified

Sea Level Indicator

12.154722

Not Specified

Sea Level Indicator

27.95

27.93131

27.9311

29.152102

Google Earth from

publication map

Terrestrial Limiting

Google Earth from

publication map

Sea Level Indicator

Google Earth from

publication map

Terrestrial Limiting

25.623122

27.925226

Google Earth from

location name

Sea Level Indicator

27.925226

Google Earth from

publication map

Sea Level Indicator

32.6975

Not Specified

Sea Level Indicator 
22.7978

22.24055

22.26111

22.2475

22.253611

22.2575

22.208888

22.263888

22.26222

22.257777

22.253611

22.256388

22.239722

22.240558

22.246388
Google Earth from publication map

Google Earth from location name

Not Specified

Not Specified

Not Specified

Terrestrial Limiting

Not Specified

Terrestrial Limiting

Not Specified

Sea Level Indicator

Not Specified

Terrestrial Limiting

Not Specified

Sea Level Indicator

Not Specified

Sea Level Indicator

Google Earth from publication map

Not Specified

Not Specified

Not Specified

Not Specified
Marine Limiting

Terrestrial Limiting

Sea Level Indicator

Terrestrial Limiting

Terrestrial Limiting 


\begin{tabular}{|c|c|c|}
\hline Type of RSL Indicator & Indicator description & $\begin{array}{l}\text { Upper limit of modern } \\
\text { analog }(m)\end{array}$ \\
\hline Lagoonal deposit & $\begin{array}{l}\text { The aated snells are iroma } \\
\text { sandy deposit interpreted } \\
\text { as lagoonal from the } \\
\text { faınal rnntent }\end{array}$ & 1 \\
\hline Lagoonal deposit & $\begin{array}{l}\text { Shell from a lagoonal } \\
\text { assemblage }\end{array}$ & 1 \\
\hline Lagoonal deposit & & 1 \\
\hline Lagoonal deposit & & 1 \\
\hline Lagoonal deposit & & 1 \\
\hline $\begin{array}{l}\text { Beach deposit or } \\
\text { beachrock }\end{array}$ & $\begin{array}{c}\text { Beach deposit on marine } \\
\text { terrace }\end{array}$ & 2 \\
\hline $\begin{array}{c}\text { The datapoint is a marine } \\
\text { or terrestrial limiting } \\
\text { indicator }\end{array}$ & $\begin{array}{l}\text { rootprınt-pearıng } \\
\text { aeolianite pre-dating } \\
\text { (underlying) beach } \\
\text { denncit nf NHN1 }\end{array}$ & \\
\hline $\begin{array}{l}\text { Beach deposit or } \\
\text { beachrock }\end{array}$ & $\begin{array}{l}\text { Coarse grained cemented } \\
\text { shelly beach facies }\end{array}$ & 0.5 \\
\hline $\begin{array}{c}\text { The datapoint is a marine } \\
\text { or terrestrial limiting } \\
\text { indicator }\end{array}$ & $\begin{array}{l}\text { Aeolıanıte overıyıng } \\
\text { earlier beach facies and } \\
\text { incised by later beach } \\
\text { farisc } \\
\text { ıransportea oyster on }\end{array}$ & \\
\hline Shore platform & $\begin{array}{l}\text { shore platform in case at } \\
4.5 \mathrm{~m} . \text { regarded as }\end{array}$ & 5 \\
\hline Lagoonal deposit & 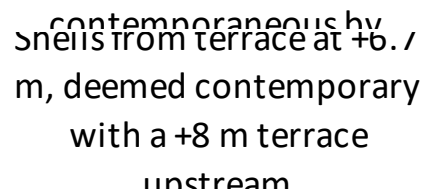 & 1 \\
\hline Lagoonal deposit & $\begin{array}{l}\text { Inctroam } \\
\text { Fine-medium bioturbated } \\
\text { sand with occasional } \\
\text { marine shells } \\
\text { rine-mealum pıoturpatea }\end{array}$ & 1 \\
\hline Lagoonal deposit & $\begin{array}{l}\text { sand with marine shells } \\
\text { overlain conformably by } \\
\text { oravel ctnrm hoarh }\end{array}$ & 1 \\
\hline $\begin{array}{l}\text { Beach deposit or } \\
\text { beachrock }\end{array}$ & $\begin{array}{l}\text { Submerged beachrock } \\
\text { from continental shelf }\end{array}$ & 0.5 \\
\hline
\end{tabular}




Beach deposit or
beachrock
The datapoint is a marine
or terrestrial limiting
indicator

Shore platform

Beach swash deposit

Shore platform

Shore platform

The datapoint is a marine or terrestrial limiting indicator

The datapoint is a marine or terrestrial limiting indicator

The datapoint is a marine or terrestrial limiting indicator

Tidal inlet facies (Western Cape, South Africa)

Tidal inlet facies (Western Cape, South Africa)

Beach swash deposit

Foreshore deposits

Tidal inlet facies (Western Cape, South Africa)

The datapoint is a marine or terrestrial limiting indicator
Beachrock on continental

shelf

tıepnant tusk In eroaea pothole on wave-cut

surface ca $6 \mathrm{~m}$ ASL. Tusk

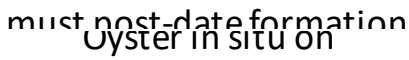

hardground/rock

platform at $4 \mathrm{~m}$.

1

Annompinnôraryoyrestor

beach facies assemblage from subtidal through

2

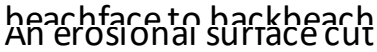

into earlier (also

presumed $5 \mathrm{e}$ ) regressive

chnroline and tonnned wivith

above adjacent

contemporary shore

$$
\text { nlatform }
$$

Corals growing on erosional terrace

Aeolianite

Aeolianite

I aal ınıet racıes (aatea)

overlain by aeolian facies

(also dated). Sea level

determined hy

Sedimentary facies

indicative of tidal inlet

depositional environment

Sandy beach berm on high energy coast

Sandy foreshore deposits overlying gravel foreshore

Lower part of tidal inlet unit

Aeolianite unit overlying tidal inlet facies apparently conformably 


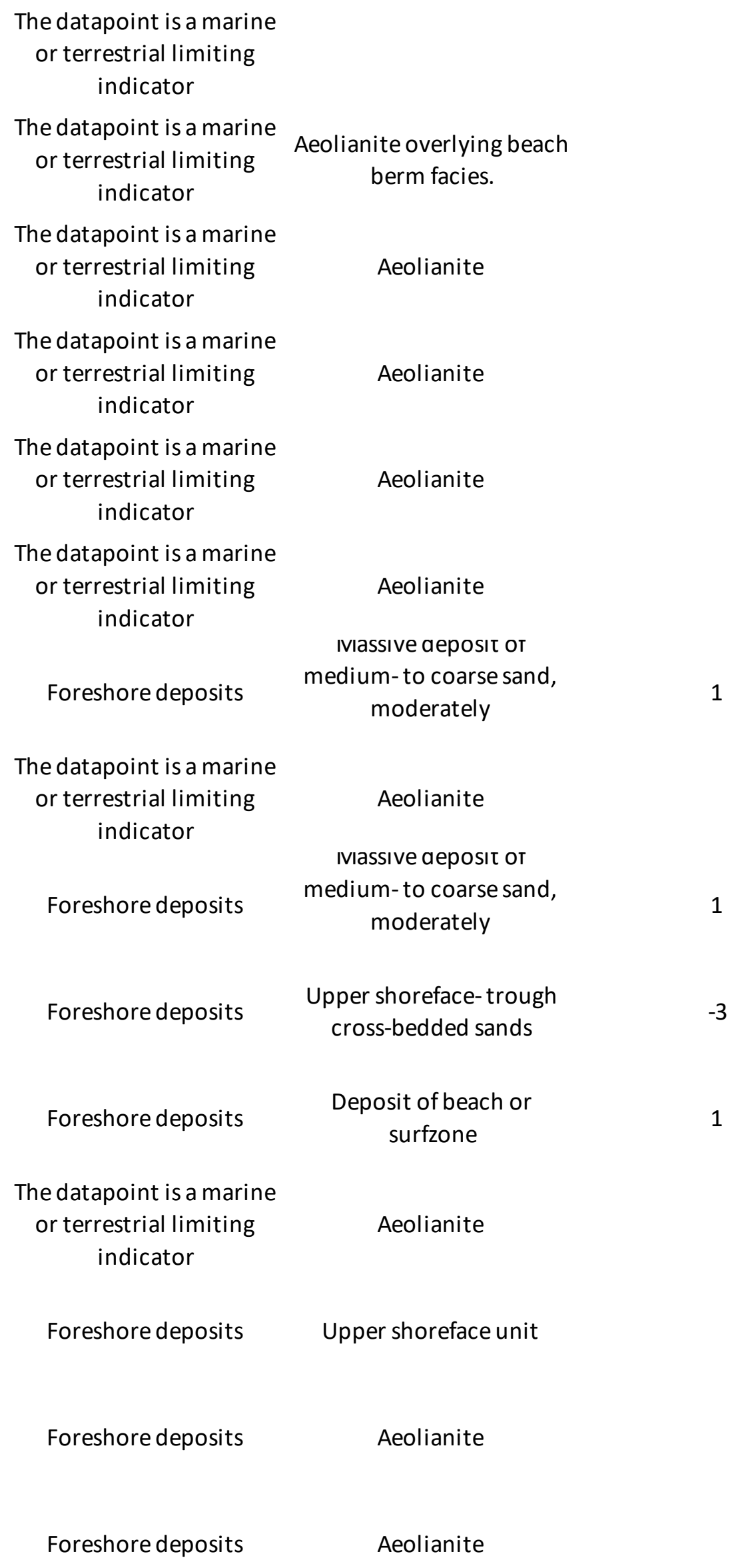


Foreshore deposits

Foreshore deposits

Foreshore deposits

Foreshore deposits

Foreshore deposits

Shoreface/foreshore contact

Lagoonal deposit

Shoreface/foreshore contact

Shoreface/foreshore contact

The datapoint is a marine or terrestrial limiting indicator
Aeolianite

Aeolianite

Aeolianite

Aeolianite

Upper shoreface

Beach-surf zone facies

1

Marginal lagoonal deposit with contemporaneous

1 aeolian deposits adjacent Assocıatea shoreface/foreshore contact provides concise

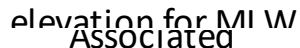
shoreface/foreshore contact provides concise elevation for MI W/

Aeolianite
$-1$

$-1$ 


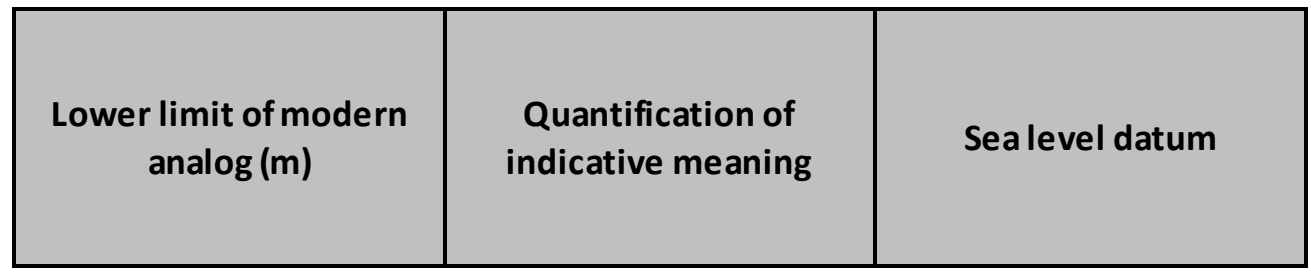

$-2$

$-2$

$-2$

$-2$

$-2$

$-2$

$-0.5$

$-3$

$-3$

$-3$

$-0.5$
Modern analog data

Modern analog data

Modern analog data

Modern analog data

Modern analog data

Modern analog data

N/A

Modern analog data

N/A

Modern analog data

Modern analog data

Modern analog data

Modern analog data

Modern analog data
Mean Sea Level / General definition

Mean Sea Level / General definition

Mean Sea Level / General definition

Mean Sea Level / General definition

Mean Sea Level / General definition

Mean Sea Level / General definition

Mean Sea Level / General definition

Mean Sea Level / General definition

Mean Sea Level / General definition

Mean Sea Level / General definition

Mean Sea Level / General definition

Mean Sea Level / General definition

Mean Sea Level / General definition

Mean Sea Level / General definition 
Mean Sea Level / General definition

1

1

$-0.5$

0

Modern analog data

N/A

N/A

N/A

$-3.5$

$-3.5$

1.5

$-3$

Modern analog data

Modern analog data

Modern analog data
Mean Sea Level / General definition

Mean Sea Level / General definition

Mean Sea Level / General definition

Mean Sea Level / General definition

Mean Sea Level / General definition

Mean Sea Level / General definition

Mean Sea Level / General definition

Mean Sea Level / General definition

Mean Sea Level / General definition

Mean Sea Level / General definition

Mean Sea Level / General definition 
Mean Sea Level / General definition

N/A

Mean Sea Level / General definition

N/A

Mean Sea Level / General definition

N/A

Mean Sea Level / General definition

N/A

Mean Sea Level / General definition

N/A

Mean Sea Level / General definition

Mean Sea Level / General definition

N/A

Mean Sea Level / General definition

Mean Sea Level / General definition

Modern analog data

Mean Sea Level / General definition

Mean Sea Level / General definition

Mean Sea Level / General definition

Mean Sea Level / General definition

Mean Sea Level / General definition

N/A

Mean Sea Level / General definition 


\begin{tabular}{|c|c|c|}
\hline & N/A & $\begin{array}{c}\text { Mean Sea Level / General } \\
\text { definition }\end{array}$ \\
\hline & N/A & $\begin{array}{c}\text { Mean Sea Level / General } \\
\text { definition }\end{array}$ \\
\hline & N/A & $\begin{array}{c}\text { Mean Sea Level / General } \\
\text { definition }\end{array}$ \\
\hline & N/A & $\begin{array}{c}\text { Mean Sea Level / General } \\
\text { definition }\end{array}$ \\
\hline & N/A & $\begin{array}{c}\text { Mean Sea Level / General } \\
\text { definition }\end{array}$ \\
\hline-3 & Modern analog data & $\begin{array}{c}\text { Mean Sea Level / General } \\
\text { definition }\end{array}$ \\
\hline-1 & Modern analog data & $\begin{array}{c}\text { Mean Sea Level / General } \\
\text { definition }\end{array}$ \\
\hline-1 & Modern analog data & Mean Low Tide (MLT) \\
\hline-1 & Modern analog data & Mean Low Tide (MLT) \\
\hline-1 & Modern analog data & Mean Low Tide (MLT) \\
\hline
\end{tabular}




\begin{tabular}{|c|c|c|}
\hline $\begin{array}{c}\text { Elevation measurement } \\
\text { technique }\end{array}$ & $\begin{array}{c}\text { Do you want to insert } \\
\text { upper and lower } \\
\text { elevation limits? }\end{array}$ & $\begin{array}{c}\text { Upper elevation of } \\
\text { indicator }(\mathrm{m})\end{array}$ \\
\hline
\end{tabular}

Not reported

Yes

12

Not reported

Yes

12

Not reported

No

Not reported

No

Not reported

No

Not reported

No

Not reported

No

Not reported

Yes

6

Not reported

No

Not reported

No

Not reported

No

Not reported

No

Not reported

No

Cross-section from

publication

No 
Cross-section from

publication

Not reported

No

Not reported

No

Total station or

Auto/hand level

No

Total station or

Auto/hand level

No

Differential GPS

No

Total station or

Auto/hand level

No

Differential GPS

No

Differential GPS

No

Total station or

Auto/hand level

No

Total station or

Auto/hand level

No

Total station or

Auto/hand level

No

Total station or

Auto/hand level

No

Total station or

Auto/hand level

No

Total station or

Auto/hand level

No 
Total station or
Auto/hand level
Total station or
Auto/hand level

Not reported

Not reported

Not reported

Not reported

Total station or

Auto/hand level

Not reported

Not reported

Not reported

Not reported

Not reported

Total station or

Auto/hand level

Total station or Auto/hand level

Total station or Auto/hand level 


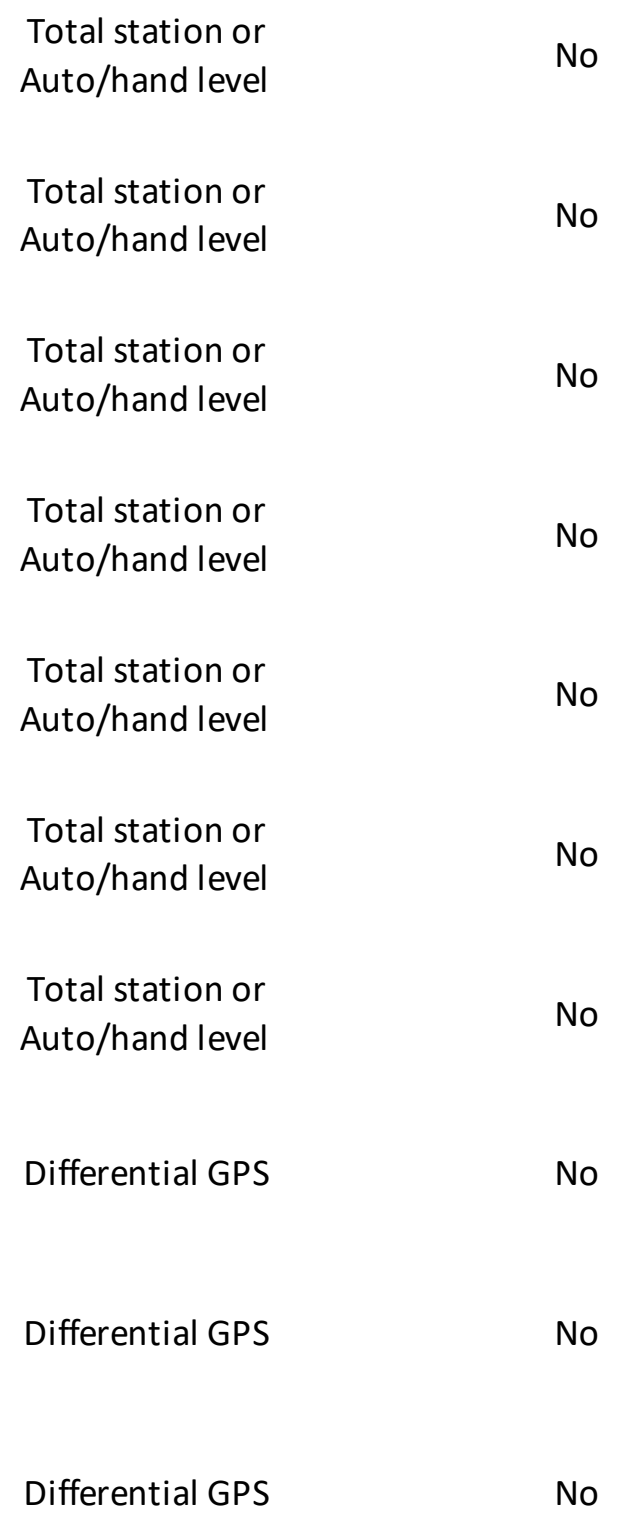




\begin{tabular}{|c|c|c|}
\hline $\begin{array}{c}\text { Lower elevation of } \\
\text { indicator }(\mathrm{m})\end{array}$ & $\begin{array}{c}\text { Upper/Lower elevation } \\
\text { measurement error }(1- \\
\text { sigma) }(\mathrm{m})\end{array}$ & $\begin{array}{c}\text { RSL indicator elevation } \\
(\mathrm{m})\end{array}$ \\
\hline
\end{tabular}


6.5

4.5

9

5.6

0.5

11.7 
6.5

10.5

$-34$

$-33$

$-30$

$-30$

0

$-35$

$-26$

$-17$

$-25$

$-38$

3.5

11.1

23.5 
20.2

13.3

10.4

9.5

6

9

1.3

5.2

5.2

7.2 


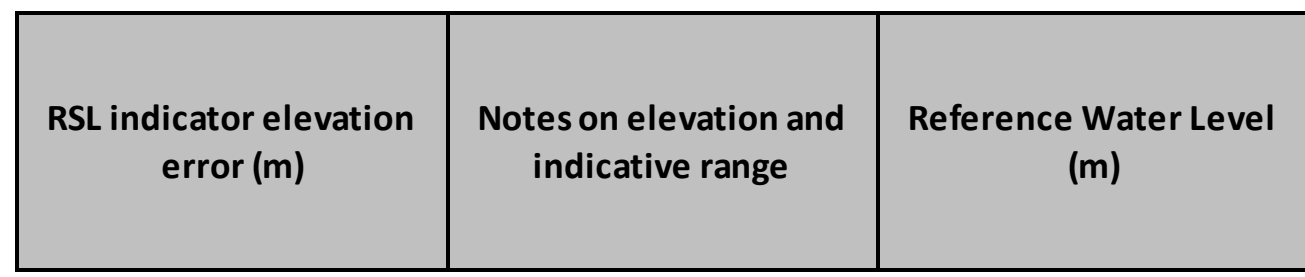

1

$-0.5$

1

$-0.5$

1

$-0.5$

1

$-0.5$

1

$-0.5$

1

0

0.1

1.75

0

elevation is or contact

between littoral

0.1

(beachrock) and aeolianite unite

1

2.5

1

$-1$

0.5

estimated measurement

error

$-1$

0.5

estimated measurement

error

$-1$ 
comparison to modern

1

beachface-backbeach elevation

comparison to modern

1 erosional platforms in

KwaZulu-Natal

ancient potholed

0.1

platform adjacent to

0

contemporary equivalent

0.1

1

1

2

This elevation marks the peak of the transgression

$-2$

0.1

$-2$

1

1.75

0.1

0

0.1

Lowermost section of tidal inlet unit

0.1 
0.1

0.1

1

1

1

1

0.1

$-1$

1

1

$-1$

1

$-11.5$

1

$-1$

1

0.05

0.05

0.05 


\begin{tabular}{|c|c|c|}
\hline Indicative Range $(\mathrm{m})$ & $\begin{array}{c}\text { Paleo Relative Sea Level } \\
(\mathrm{m})\end{array}$ & $\begin{array}{c}\text { Paleo Relative Sea Level } \\
\text { Uncertainty }(\mathrm{m})\end{array}$ \\
\hline
\end{tabular}

3

3

3

3

3

4

1

5

4

4

4

1
11.5

11.5

25.5

25.5

25.5

15

2.23

4.25

1.82

2

2.69

9

2.23

6

2.06

7.5

2.06

$-17$

2.06 
0

1

1

0

3

3

0.5

6

4
3

4.5

5

4

0.1
1.11

1.11

2.5

1.5

1.03

5.6

3

2 
4

2

0

0

0
10

1.3

6.2

6.2

0.02

8.2

0.02 


\begin{tabular}{|c|c|c|}
\hline $\begin{array}{c}\text { Is data on vertical land } \\
\text { movements } \\
\text { (independent from the } \\
\text { sea level record) } \\
\text { available? }\end{array}$ & Tectonic category & $\begin{array}{c}\text { Comments on tectonic } \\
\text { category }\end{array}$ \\
\hline
\end{tabular}

No

No

No

No

No

No

No 
No

No

No

No

No

No

No 


\begin{tabular}{|c|c|c|}
\hline $\begin{array}{c}\text { Published VLM rate } \\
(\mathrm{m} / \mathrm{ky})\end{array}$ & $\begin{array}{c}\text { Published VLM rate } \\
\text { uncertainty }(\mathrm{m} / \mathrm{ky})\end{array}$ & $\begin{array}{c}\text { Interpreted VLM rate } \\
(\mathrm{m} / \mathrm{ky})\end{array}$ \\
\hline
\end{tabular}







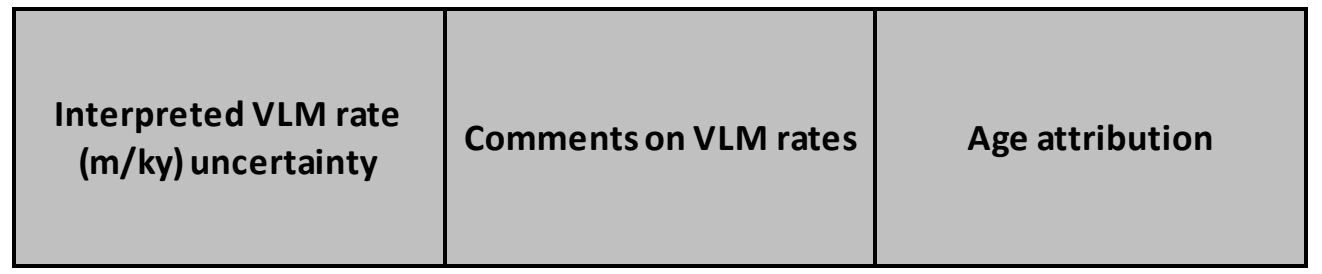

U-Series

U-Series

Luminescence

Luminescence

Luminescence

U-Series

Luminescence

Luminescence

Luminescence

U-Series

Amino Acid Racemization

Luminescence

Luminescence

U-Series 
U-Series

U-Series

U-Series

Chronostratigraphy

Chronostratigraphy

Chronostratigraphy

U-Series

U-Series

Luminescence

U-Series

Luminescence

Luminescence

Luminescence

Luminescence

Luminescence

Luminescence

Luminescence 
Luminescence

Luminescence

Luminescence

Luminescence

Luminescence

Luminescence

Luminescence

Luminescence

Luminescence

Luminescence

Luminescence

Luminescence

Luminescence

Luminescence

Luminescence 
Luminescence

Luminescence

Luminescence

Luminescence

Luminescence

Luminescence

U-Series

Luminescence

Luminescence

Luminescence

Luminescence 


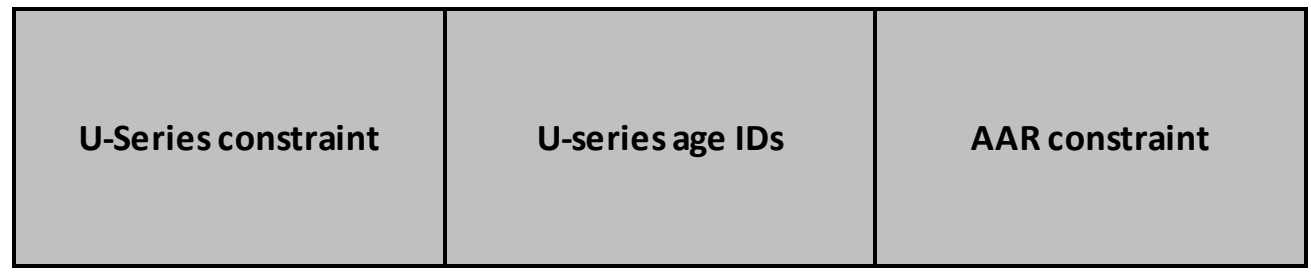

Equal to

GI84-001-001

Equal to

GI84-00-001

Equal to

GI84-003-001

Equal to

RA02-001-001

Equal to

Equal to

Equal to

Equal to

Equal to

Equal to

RA94-001-001 
Equal to

Older than

Equal to

Equal to

Equal to

Equal to
RA94-002-001

RA93-001-001

RA02-002-001

RA02-002-001

B012-001-001

B012-002-001 

Equal to

Equal to

Equal to

Equal to
R097-001-001

R097-002-001

Equal to

Equal to

Equal to 


\begin{tabular}{|c|c|c|}
\hline $\begin{array}{c}\text { Amino Acid Racemization } \\
\text { age IDs }\end{array}$ & ESR constraint & $\begin{array}{c}\text { Electro Spin Resonance } \\
\text { age IDs }\end{array}$ \\
\hline
\end{tabular}

DA80-001-001

Equal to

Equal to 


Equal to

Equal to

Equal to 


\begin{tabular}{|l|l|l|}
\hline Luminescence constraint & Luminescence age IDs & Stratigraphic constraint \\
\hline
\end{tabular}

\begin{tabular}{|c|c|}
\hline \multirow{4}{*}{ Equal to } & 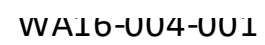 \\
\hline & WA16-003-001 \\
\hline & WA16-002-001 \\
\hline & W/ \\
\hline Equal to & WA16-006-0 \\
\hline
\end{tabular}

Equal to

WA16-007-001

Equal to

JA09-001-001

Equal to

JA09-002-001

Equal to

JA09-003-001

Equal to

WA08-001-001

Equal to 
Younger than

Younger than

Younger than

Equal to

Equal to

Equal to

Equal to

Equal to

Equal to

Equal to
B012-001-001

B012-002-001

CA10-001-001

CA10-002-001

CA10-003-001

CA10-004-001

CA10-005-001

CA10-006-001 
Equal to

Equal to

Equal to

Equal to

Equal to

Equal to

Equal to

Equal to

Equal to

Equal to

Equal to

Equal to

Equal to

Equal to

Equal to
CA10-007-001

CA10-008-001

CA14-004-001

CA14-001-001

CA14-005-001

CA14-002-001

CA14-003-001

CA14-006-001

CA14-007-001

CA14-008-001

CA14-009-001

CA14-010-001

CA18-007-001

CA18-001-001

CA18-002-001 
Equal to

Equal to

Equal to

Equal to

Equal to

Equal to

Equal to

Equal to

Equal to

Equal to
CA18-008-001

CA18-009-001

CA18-010-001

CA18-011-001

CA18-006-001

CA18-003-001

CA18-004-001

R097-003-001

R097-002-001

R097-001-001

R012-001-001

R012-002-001

R012-003-001 


\begin{tabular}{|c|c|c|}
\hline $\begin{array}{c}\text { Stratigraphic context/age } \\
\text { IDs }\end{array}$ & Other age constraint & Other age constraints IDs \\
\hline
\end{tabular}

Equal to

Equal to 
Isipingo Formation on

Durban Bluff

Isipingo Formation on

Durban Bluff

Isipingo Formation on

Durban Bluff 

Equal to

Equal to

Equal to 


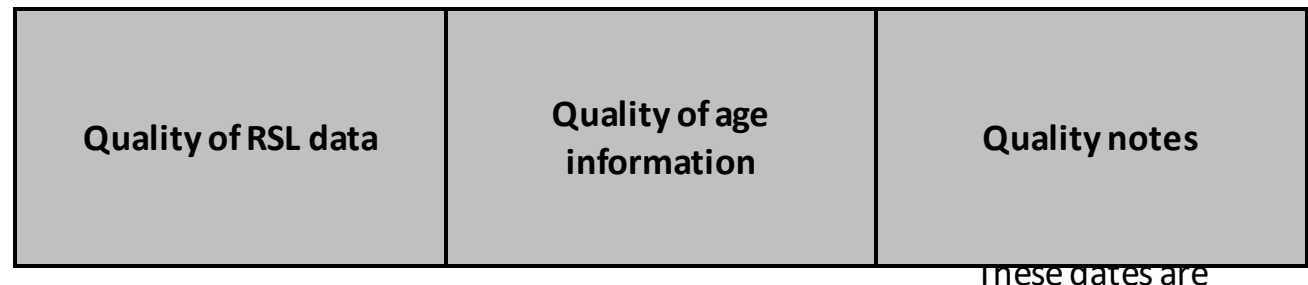

These aates are

2

1

inconsistent with later

OSL dates from higher

olevationc

1

2

3

4

3

4

3

4

1

1

1

4

3

4

1

4

1

2

2

2

3

4

uncertaınty ın ınaıcatıve

meaning but

stratigraphically

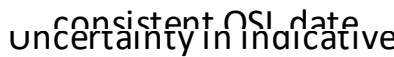

meaning of estuary

3

4

deposits. Good quality

mondern date 

only 
1

1

1

1

1

1

3

1

3

2

3

1

1

1

1
4

4

4

4

4

4

4

4

4

4

4

4

4

4

4 
1

1

1

1

1

3

3

0

0

0
4

4

4

4

4

4

3

0

0

0 


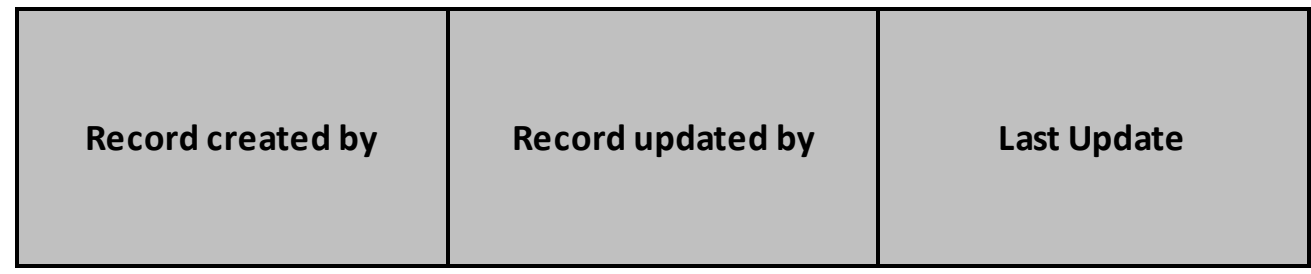

Andrew Cooper

Andrew Cooper

2020-04-08 05:16:15

Andrew Cooper

Andrew Cooper

2020-04-08 05:16:22

Andrew Cooper

Andrew Cooper

2020-04-08 05:16:37

Andrew Cooper

Andrew Cooper

2020-04-08 05:16:45

Andrew Cooper

Andrew Cooper

2020-04-08 05:17:38

Andrew Cooper

Andrew Cooper

2020-04-08 05:16:28

Andrew Cooper

Andrew Cooper

2020-04-08 05:15:32

Andrew Cooper

Andrew Cooper

2020-11-26 14:02:33

Andrew Cooper

Andrew Cooper

2020-04-08 05:15:50

Andrew Cooper

2020-04-07 09:47:55

Andrew Cooper

WALIS Admin

2020-12-16 10:26:03

Andrew Cooper

Andrew Cooper

2020-12-02 14:51:41

Andrew Cooper

Andrew Cooper

2020-12-02 14:52:00

Andrew Cooper

Andrew Cooper 
Andrew Cooper

Andrew Cooper

Andrew Cooper

Andrew Cooper

Andrew Cooper

Andrew Cooper

Andrew Cooper

Andrew Cooper

Andrew Cooper

Andrew Cooper

Andrew Cooper

Andrew Cooper

Andrew Cooper

Andrew Cooper
Andrew Cooper

2020-04-08 05:16:07

Andrew Cooper

2020-04-08 06:17:56

Andrew Cooper

2020-04-08 10:53:41

Andrew Cooper

2020-04-08 07:38:23

Andrew Cooper

2020-04-08 07:37:53

Andrew Cooper

2020-04-08 09:40:22

Andrew Cooper

2020-04-08 09:38:41

Andrew Cooper

2020-04-09 05:11:48

Andrew Cooper

2020-04-09 05:11:17

Andrew Cooper

2020-04-08 05:13:53

Andrew Cooper

2020-04-08 05:14:10

Andrew Cooper

2020-04-08 05:14:27

Andrew Cooper

2020-04-08 05:14:46

Andrew Cooper

2020-04-08 05:15:00

Andrew Cooper 
Andrew Cooper

2020-04-08 05:15:24

Andrew Cooper

Andrew Cooper

2020-04-08 05:17:47

Andrew Cooper

Andrew Cooper

2020-04-08 05:17:53

Andrew Cooper

Andrew Cooper

2020-04-08 05:17:59

Andrew Cooper

Andrew Cooper

2020-04-08 05:18:07

Andrew Cooper

Andrew Cooper

2020-04-08 05:18:13

Andrew Cooper

Andrew Cooper

2020-04-08 05:18:19

Andrew Cooper

Andrew Cooper

2020-04-08 05:18:26

Andrew Cooper

Andrew Cooper

2020-04-08 05:18:31

Andrew Cooper

Andrew Cooper

2020-04-08 05:18:38

Andrew Cooper

Andrew Cooper

2020-04-08 05:18:46

Andrew Cooper

Andrew Cooper

2020-04-06 10:24:09

Andrew Cooper

Andrew Cooper

2020-04-08 05:18:59

Andrew Cooper

Andrew Cooper

2020-04-08 05:19:10 
Andrew Cooper

Andrew Cooper

Andrew Cooper

Andrew Cooper

Andrew Cooper

Andrew Cooper
Andrew Cooper

2020-04-08 05:19:17

Andrew Cooper

2020-04-08 05:19:27

Andrew Cooper

2020-04-07 16:58:53

Andrew Cooper

2020-04-07 16:59:07

Andrew Cooper

2020-04-07 16:59:19

WALIS Admin

2021-01-21 15:57:41

Andrew Cooper

2020-04-07 16:59:45

WALIS Admin

2021-01-21 15:58:14

WALIS Admin

2021-01-21 15:58:30

WALIS Admin 
11

29

30

15

14

36

0

32 
Name of RSL indicator

Beach deposit or beachrock

Beach swash deposit

Foreshore deposits

Lagoonal deposit

Shore platform

Shoreface/foreshore contact

The datapoint is a marine or terrestrial limiting indicator

Tidal inlet facies (Western Cape, South Africa) 
Definition by Mauz et al., 2015: "Beachrocks are lithified coastal deposits that are organized in sequences of slabs with seaward inclination generally between $5^{\circ}$ and $15^{\circ}$. Definition of indicative meaning from Rovere et al., 2016.

part of the beach face located between mean sea level and foredune

Beach deposits characterized by a horizontal or gentle seawarddipping lamination.

Lagoonal deposits consist of silty and/or clayey sediments, horizontally laminated (Zecchin et al., 2004) and associated with fossils of brackish or marine water fauna. Definition of indicative meaning from Rovere et al., 2016.

Kennedy, 2015 defines shore platforms as "sub-horizontal rocky surfaces that interrupt vertical cliffs at or near sea-level". Definition of indicative meaning adapted by Rovere et al., 2016 from Kennedy, 2015.

Highest el evation of contact between cross-bedded gravelly shoreface sands and planar bedded, gently seaward dipping, foreshore sands,. Occurs at MLW.

See detailed indicator description

Coarse-grained, thickly bedded, trough cross bedding, herringbone cross bedding, multiple scours, Ophiomorpha and Skolithos trace fossils. 
(Ordinary berm + breaking depth) / 2

(Upper limit + Lower limit) / 2

Upper limit = spring tidal range $/ 2$ or $\mathrm{MHHW}$

Lower limit $=$ MSL

$(\mathrm{MHHW}$ to $\mathrm{MLLW}) / 2$

$(M L L W+$ modern Lagoon depth)/2

[Mean Higher High Water + (Breaking depth-MLLW)/2]/2

The indicator marks exactly Mean Low Water

No RWL Available

Midpoint of the Indicative range 
Ordinary berm - breaking depth

(Upper limit - Lower limit)

Upper limit = spring tidal range $/ 2$ or MHHW

Lower limit = MSL

MHHW to MLLW

MLLW to modern Lagoon depth

Mean Higher High Water - (Breaking depth-MLLW)/2

As the indicator is reported to mark exactly the MLW, the IR is zero.

No IR available

$-0.5 \mathrm{~m}$ to $-3.5 \mathrm{~m}$ with respect to MSL 
Indicator reference(s)

Mauz et al., 2015

Rovere et al., 2016

Rovere et al., 2016

Zecchin et al., 2004

Kennedy, 2015

Rovere et al., 2016

Roberts, Karkanas et al., 2012

Carr et al., 2010 
Record created by

Alessio Rovere

Deirdre Ryan

Alessio Rovere

Alessio Rovere

Alessio Rovere

Andrew Cooper

Andrew Cooper 
Record updated by

WALIS Admin

WALIS Admin

Alessio Rovere

WALIS Admin

Alessio Rovere

WALIS Admin

WALIS Admin

WALIS Admin 
2021-01-05

2021-01-05

2020-04-19

2021-01-05

2020-04-19

2021-01-21

2020-04-02

2020-12-16 
Measurement technique

Cross-section from publication

Differential GPS

Not reported

Total station or Auto/hand level 
The elevation was extracted from a published sketch/topogrpahic section.

urs posıtıons acquirea In tne tieıa ana correctea eıtner In reaı time or during post-processing with respect to the known position of a base station or a geostationary satellite system (e.g. Omnistar). Accuracy depends on satellite signal strength, distance from base station, and number of static positions acquired at the same Inration

The el evation measurement technique was not reported, most probably hand level or metered tape.

I Ital stations or I evels measure sıope alstances trom tne instrument to a particular point and triangulate relative to the $X Y Z$ coordinates of the base station. The accuracy of this process depends on how well defined the reference point and on the distance of the surveyed point from the base station. Thus, it is nereccarv to henchmark the reference ctation with a nearhy tidal 
Typical accuracy

Variable, depending on the scale of the sketch or topographic section.

$\pm 0.02 / \pm 0.08 \mathrm{~m}$, depending on survey conditions and instruments used (e.g., single-band vs dual-band receivers)

$20 \%$ of the original el evation reported added in root mean square to the sea level datum error

$\pm 0.1 / \pm 0.2 \mathrm{~m}$ for total station $\pm 0.2 / \pm 0.4 \mathrm{~m}$ for hand level 
Record created by

Alessio Rovere

Alessio Rovere

Alessio Rovere

Alessio Rovere 
Record updated by

Alessio Rovere

Alessio Rovere

Alessio Rovere 
2020-04-19 14:13:26

2020-04-19 14:10:31

2020-01-31 11:06:03

2019-04-04 04:49:38 
4

1 
Measurement technique

Differential GPS

Google Earth from location name

Google Earth from publication map

Not Specified 
GPS receiving differential corrections from satellite or on-land base stations.

The location name was geocoded in Google Earth.

Location data is presented in a map within the publication, coordinates were extracted by georeferencing the map into Google Earth and digitizing points of interest.

The original study does not specify how the location of sites/samples was measured. 
Typical accuracy

Few centimeters, depending on accuracy.

Depending on how precise is the Google Earth geocoding.

Up to tens of meters, depending on how precise is the originally published map.

Not available or not reported 
Record created by

Alessio Rovere

Alessio Rovere

Alessio Rovere

Alessio Rovere 
Record updated by

Alessio Rovere

Alessio Rovere

Alessio Rovere

Alessio Rovere 
2020-05-08 03:23:48

2020-04-19 13:51:23

2020-04-19 13:50:54

2020-04-19 14:02:33 
1

13 
Mean Low Tide (MLT)

Mean Sea Level / General definition

Not reported 
Datum description

From tideschart.com: "The Mean Low Tide (or Mean Low Water) is the average height of all low tides in a given place, deriving from a long series of observations (NTDE) of all levels of low tide in that spot."

General definition of MSL, with no indications on the datum to which it is referred to.

The sea level datum is not reported and impossible to derive from metadata. 
Depends

A datum uncertainty may be established on a case-by-case basis.

N/A 
Record created by

Alessio Rovere

Alessio Rovere

Alessio Rovere 
Alessio Rovere 
2020-04-07 14:33:32

2020-04-19 14:25:12

2020-04-07 14:27:03 


\begin{tabular}{|c|c|c|}
\hline WALIS U-Series ID & Is this datapoint public? & Material type \\
\hline 1684 & 0 & Mollusk or algae \\
\hline 1685 & 0 & Mollusk or algae \\
\hline 793 & 0 & Mollusk or algae \\
\hline 792 & 0 & Mollusk or algae \\
\hline 794 & 0 & Mollusk or algae \\
\hline 1682 & 0 & Mollusk or algae \\
\hline 1683 & 0 & Mollusk or algae \\
\hline 1681 & 0 & Mollusk or algae \\
\hline 790 & 0 & Mollusk or algae \\
\hline 791 & 0 & Mollusk or algae \\
\hline 806 & 0 & Mollusk or algae \\
\hline 808 & 0 & Mollusk or algae \\
\hline
\end{tabular}




\begin{tabular}{|c|c|c|}
\hline Details on dated material & Type of spectrometry & Reference(s) \\
\hline Aeolianite cement & Mass spectrometer & Bosman, 2012 \\
\hline \multirow[t]{4}{*}{ Aeolianite cement } & Mass spectrometer & Bosman, 2012 \\
\hline & Alpha & Giresse et al., 1984 \\
\hline & Alpha & Giresse et al., 1984 \\
\hline & Alpha & Giresse et al., 1984 \\
\hline Oyster shell (transported) & Alpha & Ramsay and Cooper, 2002 \\
\hline $\begin{array}{c}\text { Oyster on } \\
\text { hardground/rock }\end{array}$ & Alpha & Ramsay and Cooper, 2002 \\
\hline $\begin{array}{c}\text { Elephant tusk in pothole } \\
\text { on marine terrace }\end{array}$ & Alpha & Ramsay et al. 1993 \\
\hline Beachrock & Mass spectrometer & Ramsay, 1994 \\
\hline Beachrock & Mass spectrometer & Ramsay, 1994 \\
\hline $\begin{array}{l}\text { Hominid footprint- } \\
\text { bearing lagoonal } \\
\text { sediments }\end{array}$ & Alpha & Roberts and Berger 1997 \\
\hline $\begin{array}{l}\text { Hominid footprint- } \\
\text { bearing lagoonal } \\
\text { sediments }\end{array}$ & Alpha & Roberts and Berger 1997 \\
\hline
\end{tabular}




\begin{tabular}{|c|c|c|}
\hline GSN & Sample ID & Analysis ID \\
\hline & B012-001 & BO12-001-001 \\
\hline & BO12-002 & BO12-002-001 \\
\hline & G184-002 & GI84-00-001 \\
\hline & GI84-001 & GI84-001-001 \\
\hline & G184-003 & GI84-003-001 \\
\hline & RA02-001 & RA02-001-001 \\
\hline & RA02-002 & RA02-002-001 \\
\hline & RA93-001 & RA93-001-001 \\
\hline & RA94-001 & RA94-001-001 \\
\hline & RA94-002 & RA94-002-001 \\
\hline & R097-001 & R097-001-001 \\
\hline & R097-002 & R097-002-001 \\
\hline
\end{tabular}




\begin{tabular}{|c|c|c|}
\hline Reported ID & Date of analysis & Accepted? \\
\hline GC-4 & & Yes \\
\hline GC-7 & & Yes \\
\hline AN57-2 & & Yes \\
\hline AN57-1 & & Yes \\
\hline AN40-2 & & Yes \\
\hline PTA-U568 & & Yes \\
\hline PTA-U565 & & Yes \\
\hline U415 & & Yes \\
\hline PTA-U-435 & & Yes \\
\hline PTA-U-487 & & Yes \\
\hline RB97-04 & & Yes \\
\hline RB97-05 & & Yes \\
\hline
\end{tabular}




\begin{tabular}{|l|l|l|}
\hline Reason for rejection & Screening & $\begin{array}{c}\text { Latitude (decimal } \\
\text { degrees) }\end{array}$ \\
\hline
\end{tabular}

$-30.25$

$-30.25$

$-12.620277$

$-12.620277$

$-15.133055$

$-31.987624$

$-27.878164$

$-29.98444$

$-27.50777$

$-27.50777$

$-33.152777$

$-33.152777$ 


\begin{tabular}{|c|c|c|}
\hline $\begin{array}{c}\text { Longitude (decimal } \\
\text { degrees) }\end{array}$ & Reported Latitude & Reported Longitude \\
\hline
\end{tabular}

30.816667

30.816667

13.243611

13.243611

12.154722

29.152102

32.3299

30.96545

32.6975

32.6974

18.038333

18.038333 


\begin{tabular}{|c|c|c|}
\hline Are Lat/Long estimated? & $\begin{array}{c}\text { Comments on geographic } \\
\text { coordinates }\end{array}$ & $\begin{array}{c}\text { Original elevation datum } \\
\text { used }\end{array}$ \\
\hline Yes & & Not reported \\
\hline Yes & & Not reported \\
\hline Yes & & Not reported \\
\hline Yes & & Not reported \\
\hline Yes & & Not reported \\
\hline Yes & & Not reported \\
\hline Yes & & Not reported \\
\hline Yes & & Not reported \\
\hline Yes & & Not reported \\
\hline Yes & & Not reported \\
\hline & & Not reported \\
\hline Yes & & Not reported \\
\hline
\end{tabular}




\begin{tabular}{|c|c|c|}
\hline $\begin{array}{c}\text { Elevation measurement } \\
\text { method }\end{array}$ & Reported elevation $(\mathrm{m})$ & $\begin{array}{c}\text { Reported elevation } \\
\text { uncertainty }(\mathrm{m})\end{array}$ \\
\hline
\end{tabular}

Not reported

$-15$

1

Not reported

1

Not reported

10-12

N/A

Not reported

$10-12$

N/A

Not reported

15

N/A

Not reported

4.5

1

Not reported

4

0.1

Not reported

6

1

Cross-section from

publication

N/A

Not reported

$-44$

2

Not reported

1.7

0.1

Not reported

1.7

0.1 


\begin{tabular}{|c|c|c|}
\hline Elevation above MSL (m) & $\begin{array}{c}\text { Elevation uncertainty } \\
\text { used }(\mathbf{m})\end{array}$ & Elevation comments \\
N/A & N/A \\
N/A & N/A \\
N/A & N/A \\
N/A & N/A \\
N/A & N/A \\
N/A & N/A & \\
N/A & & \\
N/A & &
\end{tabular}




\begin{tabular}{|l|l|l|}
\hline Terrace ID & Facies description & Reported as in situ? \\
\hline
\end{tabular}

No

Beachrock 


\begin{tabular}{|c|c|c|}
\hline $\begin{array}{c}\text { Reported as in growth } \\
\text { position? }\end{array}$ & $\begin{array}{c}\text { Taxa information (as } \\
\text { reported) }\end{array}$ & Family \\
\hline
\end{tabular}




\begin{tabular}{|l|l|l|}
\hline Genus & Species & $\mathrm{Pa} /$ Th age? \\
\hline
\end{tabular}

Yes

Yes

No

No 


\begin{tabular}{|c|c|c|}
\hline 14C age? & Instrument & Decay constants \\
\hline & TIMS & No Info \\
\hline & TIMS & No Info \\
\hline & Not reported & No Info \\
\hline & Not reported & No Info \\
\hline & Not reported & No Info \\
\hline & Not reported & No Info \\
\hline & Not reported & No Info \\
\hline & Not reported & No Info \\
\hline & Not reported & No Info \\
\hline & Not reported & No Info \\
\hline No & Not reported & No Info \\
\hline No & Not reported & No Info \\
\hline
\end{tabular}




\begin{tabular}{|c|c|c|}
\hline $\begin{array}{c}\text { Comments on decay } \\
\text { constants }\end{array}$ & $\begin{array}{c}\text { Calibration method for } \\
\text { 230Th/238U ratio }\end{array}$ & $\begin{array}{c}\text { Calibration method for } \\
234 U / 238 U \text { ratio }\end{array}$ \\
\hline
\end{tabular}

SE

SE

SE

SE

SE

SE

SE

SE

SE

SE

SE

SE
SE

SE

SE

SE

SE

SE

SE

SE

SE

SE

SE

SE 
Comments on spike calibration
Published \% calcite

Interpreted \% calcite 


\begin{tabular}{|c|c|c|}
\hline $\begin{array}{c}\text { [234Th/238U]ACT } \\
\text { backcalculated? }\end{array}$ & {$[232 \mathrm{Th}](\mathrm{ppb})$} & $\begin{array}{c}\text { [232Th] (ppb) uncertainty } \\
( \pm 2 \sigma)\end{array}$ \\
\hline
\end{tabular}




\begin{tabular}{|c|c|c|}
\hline$[238 \mathrm{U}](\mathrm{ppm})$ & $\begin{array}{c}{[238 \mathrm{U}](\mathrm{ppm}) \text { uncertainty }} \\
( \pm 2 \sigma)\end{array}$ & $\begin{array}{c}\text { Atomic ratio } \\
(232 \mathrm{Th} / 238 \mathrm{U}) * 10^{\wedge} 5\end{array}$ \\
\hline
\end{tabular}




\begin{tabular}{|c|c|c|}
\hline Initial 230Th/232Th & [230Th/232Th]ACT & $\begin{array}{c}\text { [230Th/232Th]ACT } \\
\text { uncertainty }( \pm 2 \sigma)\end{array}$ \\
\hline
\end{tabular}




\begin{tabular}{|l|c|c|}
\hline$[232 \mathrm{Th} / 238 \mathrm{U}] \mathrm{ACT}$ & $\begin{array}{c}{[232 \mathrm{Th} / 238 \mathrm{U}] \mathrm{ACT}} \\
\text { uncertainty }( \pm 2 \sigma)\end{array}$ & [230Th/234U]ACT \\
\hline
\end{tabular}




\begin{tabular}{|c|c|c|}
\hline $\begin{array}{c}\text { Correction factor for } \\
230 \mathrm{Th} / 238 \mathrm{U}\end{array}$ & $\begin{array}{c}\text { Correction factor for } \\
230 \mathrm{Th} / 238 \mathrm{U} \text { uncertainty } \\
( \pm 2 \sigma)\end{array}$ & $\begin{array}{c}\text { Correction factor for } \\
234 \mathrm{U} / 238 \mathrm{U}\end{array}$ \\
\hline
\end{tabular}




\begin{tabular}{|c|c|c|}
\hline $\begin{array}{c}\text { Correction factor for } \\
234 \mathrm{U} / 238 \mathrm{U} \text { uncertainty } \\
( \pm 2 \sigma)\end{array}$ & Comments & $\begin{array}{c}\text { Recalculated } \\
{[230 \mathrm{Th} / 238 \mathrm{U}] \mathrm{ACT}}\end{array}$ \\
\hline
\end{tabular}




\begin{tabular}{|c|c|c|}
\hline $\begin{array}{c}\text { Recalculated } \\
{[230 \mathrm{Th} / 238 \mathrm{U}] \mathrm{ACT}} \\
\text { uncertainty }( \pm 2 \sigma)\end{array}$ & $\begin{array}{c}\text { Recalculated } \\
{[234 U / 238 U] A C T}\end{array}$ & $\begin{array}{c}\text { Recalculated } \\
{[234 U / 238 U] A C T} \\
\text { uncertainty }( \pm 2 \sigma)\end{array}$ \\
\hline
\end{tabular}




\begin{tabular}{|c|c|c|}
\hline $\begin{array}{c}\text { Age is } \\
\text { Older/Equal/Younger } \\
\text { than }\end{array}$ & Marine Isotopic Stage & Age determination \\
\hline Equal to & MIS 5 & Error 6812 \\
\hline Equal to & MIS 5 & 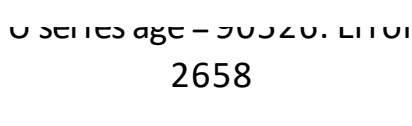 \\
\hline & AIC 5 & Th date $=107+/-6 \mathrm{ka}$ \\
\hline Lyuar & & $\begin{array}{l}\text { Pa date }=136+28,-17 \\
\text { Th Age is } 92+/-3 \mathrm{ka}\end{array}$ \\
\hline Equal to & MIS 5 & $\begin{array}{l}\text { Pa age is } 91+/-6 \mathrm{ka} \\
\text { Th age }=133+/-10 \mathrm{ka}\end{array}$ \\
\hline Equal to & MIS 5 & Pa Age $=174+x-80$ \\
\hline Equal to & MIS 5 & $\begin{array}{l}\text { Oyster shell deposited on } \\
\text { top of } 4.5 \mathrm{~m} \text { terrace in } \\
\text { cave. Regarded as near }\end{array}$ \\
\hline Equal to & MIS 5 & $\mathrm{U} / \mathrm{Th}=95.7+/-4 \mathrm{ka}$ \\
\hline Equal to & MIS 5 & Age $=112+/-23 \mathrm{ka}$ \\
\hline Equal to & MIS 5 & $84,000+/-3000$ \\
\hline Equal to & MIS 5 & $117,000+/-7000$ \\
\hline Equal to & MIS 5 & $\mathrm{U} / \mathrm{Th}=103+/-7 \mathrm{ka}$ \\
\hline Equal to & MIS 5 & $\mathrm{U} / \mathrm{Th}=102+/-7 \mathrm{ka}$ \\
\hline
\end{tabular}




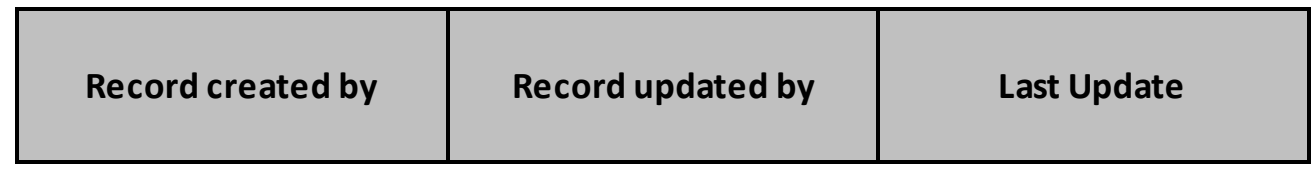

Andrew Cooper

WALIS Admin

2020-12-16 11:03:02

Andrew Cooper

WALIS Admin

2020-12-16 11:03:21

Andrew Cooper

WALIS Admin

2020-12-16 11:01:49

Andrew Cooper

WALIS Admin

2020-12-16 11:01:12

Andrew Cooper

WALIS Admin

2020-12-16 11:02:09

Andrew Cooper

WALIS Admin

2020-12-16 11:05:22

Andrew Cooper

Andrew Cooper

2020-12-16 11:05:48

Andrew Cooper

Andrew Cooper

2020-12-16 11:07:49

Andrew Cooper

Andrew Cooper

2020-12-16 11:04:23

Andrew Cooper

WALIS Admin

2020-12-16 11:04:49

Andrew Cooper

Andrew Cooper

2020-12-16 11:06:40

Andrew Cooper

Andrew Cooper 


\begin{tabular}{|l|l|l|}
\hline WALIS AAR ID & Is this datapoint public? & Reference(s) \\
\hline
\end{tabular}

128

Davies, 1980 


\begin{tabular}{|l|l|l|}
\hline IGSN & Sample ID & Analysis ID \\
\hline
\end{tabular}

DA80-001

DA80-001-001 


\begin{tabular}{|l|l|l|}
\hline Reported ID & Date of analysis & Laboratory name \\
\hline
\end{tabular}

PE-557

University of Colorado 


\begin{tabular}{|l|l|l|}
\hline Sample Type & Taxonomy & Sample position \\
\hline
\end{tabular}

Estuarine mollusc 


\begin{tabular}{|l|l|l|}
\hline Whole-Rock? & Grain Size $(\mu \mathrm{m})$ & Carbonate (\%) \\
\hline
\end{tabular}

No 


\begin{tabular}{|c|c|c|}
\hline Fraction $(\mu \mathrm{m})$ & $\begin{array}{c}\text { Notes and comments on } \\
\text { sample quality }\end{array}$ & Collection Context \\
\hline
\end{tabular}




\begin{tabular}{|l|l|l|}
\hline Depth of burial $(\mathrm{m})$ & Accepted? & Reason for rejection \\
\hline
\end{tabular}

unknown

Yes 


\begin{tabular}{|l|l|l|}
\hline Reported Latitude & Reported Longitude & $\begin{array}{c}\text { Latitude (decimal } \\
\text { degrees) }\end{array}$ \\
\hline
\end{tabular}

$-33.87108$ 


\begin{tabular}{|c|c|c|}
\hline $\begin{array}{c}\text { Longitude (decimal } \\
\text { degrees) }\end{array}$ & Are Lat/Long estimated? & $\begin{array}{c}\text { Original elevation datum } \\
\text { used }\end{array}$ \\
\hline
\end{tabular}

25.623122

Yes 


\begin{tabular}{|c|c|c|}
\hline $\begin{array}{c}\text { Elevation measurement } \\
\text { method }\end{array}$ & Reported elevation $(\mathrm{m})$ & $\begin{array}{c}\text { Reported elevation } \\
\text { uncertainty }(\mathrm{m})\end{array}$ \\
\hline
\end{tabular}

6.5

N/A 


\begin{tabular}{|l|c|c|}
\hline Elevation above MSL $(\mathrm{m})$ & $\begin{array}{c}\text { Elevation uncertainty } \\
\text { used }(\mathrm{m})\end{array}$ & Elevation comments \\
\hline
\end{tabular}




\begin{tabular}{|c|c|c|}
\hline $\begin{array}{c}\text { Age is } \\
\text { Older/Equal/Younger } \\
\text { than }\end{array}$ & Marine Isotopic Stage & $\begin{array}{c}\text { Comments/details on } \\
\text { MIS designation }\end{array}$ \\
Equal to & MIS 5 & $\begin{array}{c}\text { From Davies (1980) } \\
\text { "PE-557, Deal Party, bar at } \\
\text { mouth of Swartkops River, }\end{array}$
\end{tabular}




\begin{tabular}{|c|c|c|}
\hline Reported age (ka) & $\begin{array}{c}\text { Reported age uncertainty } \\
( \pm 2 \sigma)(\mathrm{ka})\end{array}$ & $\begin{array}{c}\text { Current Annual Mean } \\
\text { Temperature }\left({ }^{\circ} \mathrm{C}\right)\end{array}$ \\
\hline
\end{tabular}




\begin{tabular}{|c|c|c|}
\hline Preparation procedure & $\begin{array}{c}\text { Method of } \\
\text { chromatography }\end{array}$ & $\begin{array}{c}\text { Stationary phase of the } \\
\text { column }\end{array}$ \\
\hline
\end{tabular}

unknown 


\begin{tabular}{|c|c|c|}
\hline $\begin{array}{c}\text { Number of replicates } \\
\text { (samples) }\end{array}$ & $\begin{array}{c}\text { Number of sample } \\
\text { replicates (injections) }\end{array}$ & Ratio type \\
\hline
\end{tabular}




\begin{tabular}{|c|c|c|}
\hline $\begin{array}{c}\text { Pyrolysis experiment } \\
\text { available }\end{array}$ & Level of uncertainty & Aspartic \\
\hline
\end{tabular}

1-sigma 


\begin{tabular}{|l|l|l|}
\hline Serine & Serine uncertainty & Alanine \\
\hline
\end{tabular}




\begin{tabular}{|l|l|l|}
\hline$* \mathrm{~A} / \mathrm{I}$ & $* \mathrm{~A} /$ I uncertainty & Leucine \\
\hline
\end{tabular}




\begin{tabular}{|l|l|l|}
\hline Leucine uncertainty & Notes/Comments & $\begin{array}{c}\text { Independent age } \\
\text { constraints available? }\end{array}$ \\
\hline
\end{tabular}

No 


\begin{tabular}{|c|c|c|}
\hline $\begin{array}{c}\text { Calibration data } \\
\text { available? }\end{array}$ & $\begin{array}{c}\text { Are free AAR ratios } \\
\text { available? }\end{array}$ & $\begin{array}{c}\text { Level of uncertainty free } \\
\text { AAR }\end{array}$ \\
\hline
\end{tabular}

No

No

N/A 


\begin{tabular}{|c|c|c|}
\hline Aspartic (free AAR) & $\begin{array}{c}\text { Aspartic uncertainty (free } \\
\text { AAR) }\end{array}$ & Glutamic (free AAR) \\
\hline
\end{tabular}


Glutamic uncertainty (free AAR)
Serine (free AAR)
Serine uncertainty (free AAR) 


\begin{tabular}{|c|c|c|}
\hline Alanine (free AAR) & $\begin{array}{c}\text { Alanine uncertainty (free } \\
\text { AAR) }\end{array}$ & Valine (free AAR) \\
\hline
\end{tabular}




\begin{tabular}{|c|c|c|}
\hline $\begin{array}{c}\text { Valine uncertainty (free } \\
\text { AAR) }\end{array}$ & $*$ A/I (free AAR) & *A/I uncertainty (free \\
\hline
\end{tabular}




\begin{tabular}{|c|c|c|}
\hline Leucine (free AAR) & $\begin{array}{c}\text { Leucine uncertainty (free } \\
\text { AAR) }\end{array}$ & $\begin{array}{c}\text { Notes/Comments free } \\
\text { AAR }\end{array}$ \\
\hline
\end{tabular}




\begin{tabular}{|c|c|c|}
\hline $\begin{array}{c}\text { Calibration } \mathrm{D} / \mathrm{L} \text { value } \\
\text { uncertainty }( \pm 1 \sigma)\end{array}$ & Calibration age (ka) & $\begin{array}{c}\text { Calibration age (ka) } \\
\text { uncertainty }( \pm 1 \sigma)\end{array}$ \\
\hline
\end{tabular}




\begin{tabular}{|l|l|l|}
\hline Independent age choice & Independent age method & $\begin{array}{c}\text { Independent age } \\
\text { laboratory }\end{array}$ \\
\hline
\end{tabular}




\begin{tabular}{|c|l|l|}
\hline $\begin{array}{c}\text { Independent age } \\
\text { laboratory ID }\end{array}$ & Independent age result & $\begin{array}{c}\text { Independent age } \\
\text { reference }\end{array}$ \\
\hline
\end{tabular}




\begin{tabular}{|l|l|l|}
\hline U-Series age constraint & ESR age constraint & $\begin{array}{c}\text { Luminescence age } \\
\text { constraint }\end{array}$ \\
\hline
\end{tabular}




\begin{tabular}{|c|c|c|}
\hline $\begin{array}{c}\text { Stratigraphic age } \\
\text { constraint }\end{array}$ & Record created by & Record updated by \\
\hline
\end{tabular}

Andrew Cooper

WALIS Admin 


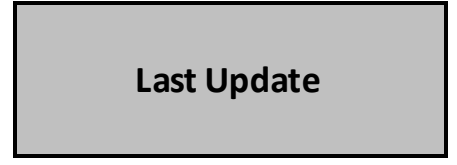

2020-12-16 11:08:52 


\begin{tabular}{|c|c|c|}
\hline WALIS LUM ID & Is this datapoint public? & Luminescence method \\
\hline 287 & & OSL \\
\hline 166 & 1 & OSL \\
\hline 167 & 1 & OSL \\
\hline 100 & 1 & OSL \\
\hline 101 & 1 & OSL \\
\hline 102 & 1 & OSL \\
\hline 103 & 1 & OSL \\
\hline 104 & 1 & OSL \\
\hline 105 & 1 & OSL \\
\hline 106 & 1 & OSL \\
\hline 107 & 1 & OSL \\
\hline 122 & 1 & OSL \\
\hline 124 & 1 & OSL \\
\hline 125 & 1 & OSL \\
\hline 121 & 1 & OSL \\
\hline 123 & 1 & OSL \\
\hline
\end{tabular}


OSL 
OSL 


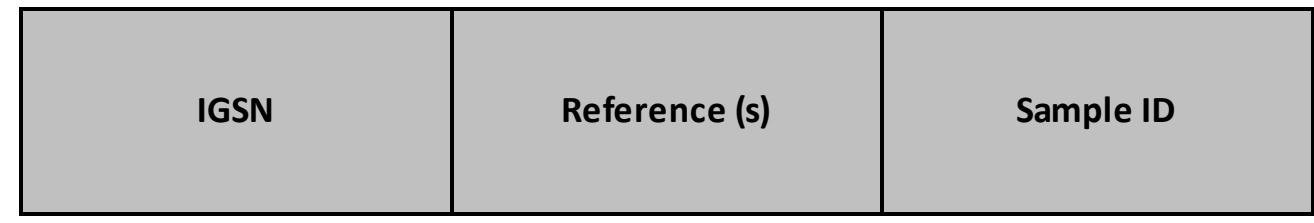

Wang et al., 2008

SAMPLE2

Bosman, 2012

B012-001

Bosman, 2012

B012-002

Carr et al., 2010

CA10-001

Carr et al., 2010

CA10-002

Carr et al., 2010

CA10-003

Carr et al., 2010

CA10-004

Carr et al., 2010

CA10-005

Carr et al., 2010

CA10-006

Carr et al., 2010

CA10-007

Carr et al., 2010

CA10-008

Cawthra 2014

CA14-001

Cawthra 2014

CA14-002

Cawthra 2014

CA14-003

Cawthra 2014

CA14-004

Cawthra 2014

CA14-005 
Roberts, Karkanas et al., 2012

R012-001

Roberts, Karkanas et al., 2012

R012-002

Roberts, Karkanas et al., 2012

R012-003

Roberts and Berger 1997

R097-001

Roberts and Berger 1997

R097-002

Roberts and Berger 1997

R097-003

Roberts and Berger 1997

R097-004

Wang et al., 2008

WA08-001

Walker et al., 2016

WA16-001

Walker et al., 2016

WA16-002

Walker et al., 2016

WA16-003

Walker et al., 2016

WA16-004

Walker et al., 2016

WA16-005

Walker et al., 2016

WA16-006

Walker et al., 2016

WA16-007 


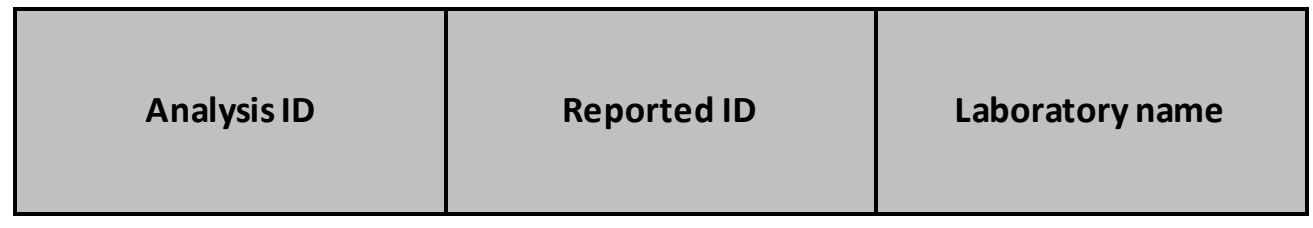

1

B012-001-001

B012-002-001

CA10-001-001

CA10-002-001

CA10-003-001

CA10-004-001

CA10-005-001

CA10-006-001

CA10-007-001

CA10-008-001

CA14-001-001

CA14-002-001

CA14-003-001

CA14-004-001

CA14-005-001
Sample2

GC-4

GC-7

UoW-234

SHFD07072

UOW-232

SHFD05019

SHFD04288

SHFD05038

UOW-235

UOW-233

HC26

Wollongong

HC36

Wollongong

$\mathrm{HCSH}$

Wollongong

HC30

Wollongong

HC35

Wollongong 


\begin{tabular}{|c|c|c|}
\hline CA14-006-001 & HC29 & Wollongong \\
\hline CA14-007-001 & $\mathrm{HC} 40$ & Wollongong \\
\hline CA14-008-001 & $\mathrm{HC} 32$ & Wollongong \\
\hline CA14-009-001 & HC39 & Wollongong \\
\hline CA14-010-001 & $\mathrm{HC} 24$ & Wollongong \\
\hline CA18-001-001 & 142832 & Wollongong \\
\hline CA18-002-001 & 157206 & \\
\hline CA18-003-001 & 142831 & Wollongong \\
\hline CA18-004-001 & 142830 & Wollongong \\
\hline CA18-006-001 & 142829 & Wollongong \\
\hline CA18-007-001 & 142833 & Wollongong \\
\hline CA18-008-001 & 157205 & Wollongong \\
\hline CA18-009-001 & 157204 & Wollongon \\
\hline CA18-010-001 & 157203 & Wollongong \\
\hline CA18-011-001 & 157202 & Wollongong \\
\hline JA09-001-001 & NN1 & \\
\hline JA09-002-001 & NHN1 & \\
\hline
\end{tabular}


JA09-003-001

NHN2

R012-001-001

Dana7

RO12-002-001

Dana6

R012-003-001

46882

R097-001-001

RB97-01

R097-002-001

RB97-02

R097-003-001

RB97-03

R097-004-001

RB97-03

WA08-001-001

Sample1

WA16-001-001

BNG10-6

WA16-002-001

BNG10-7

WA16-003-001

BNG10-8

WA16-004-001

BNG10-9

WA16-005-001

BNG10-10

WA16-006-001

BNG10-11

WA16-007-001

BNG10-12 


\begin{tabular}{|l|l|l|}
\hline Mineral type & Grain size & Date of analysis \\
\hline
\end{tabular}






\begin{tabular}{|l|l|l|}
\hline Accepted? & Reason for rejection & Reported Latitude \\
\hline
\end{tabular}

Yes

Yes

Yes

Yes

Yes

Yes

Yes

Yes

Yes

Yes

Yes

Yes

Yes

Yes

Yes

Yes 
Yes

Yes

Yes

Yes

Yes

Yes

Yes

Yes

Yes

Yes

Yes

Yes

Yes

Yes

Yes

Yes

Yes 
Yes

Yes

Yes

Yes

Yes

"regarded as unreliable

No

because of the lower dose

rate involved"

Yes

Yes

Yes

Yes

Yes

Yes

Yes

Yes

Yes

Yes 


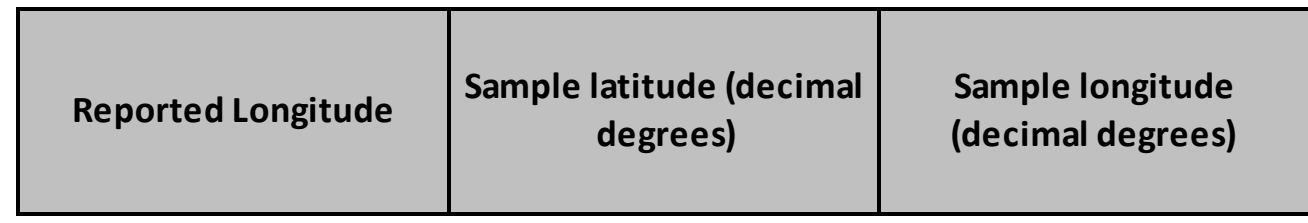

$-33.006137$

27.925226

$-30.25$

30.816667

$-30.25$

30.816667

$-34.0325$

22.7977

$-34.0325$

22.7977

$-34.05611$

22.240555

$-34.81722$

20.0275

$-34.0325$

22.7978

$-34.0325$

22.7977

$-34.0325$

22.7978

$-34.056111$

22.24055

$-34.090833$

22.2475

$-34.081666$

22.2575

$-34.064722$

22.208888

$-34.088055$

22.26111

$-34.083833$

22.253611 
$-34.089444$

$-34.073055$

$-34.068888$

$-34.078055$

$-34.1025$

$-34.056111$

$-34.055833$

$-34.056111$

$-34.056111$

$-34.055833$

$-34.056388$

$-34.055833$

$-34.055833$

$-34.055833$

$-34.055833$

$-32.994722$

$-33.006327$
22.23888

22.26222

22.25777

22.253611

22.256388

22.240558

22.246388

22.240558

22.240558

22.246388

22.239722

22.246388

22.246388

22.246388

22.246388

27.95

27.93131 


\begin{tabular}{|c|c|}
\hline-33.0225 & 27.9311 \\
\hline-34.20763 & 22.029954 \\
\hline-34.20763 & 22.029954 \\
\hline-34.20763 & 22.029954 \\
\hline-33.152777 & 18.038333 \\
\hline-33.152777 & 18.038333 \\
\hline-33.152777 & 18.038333 \\
\hline-33.152777 & 18.038333 \\
\hline-33.006137 & 27.925226 \\
\hline-12.61 & 13.365 \\
\hline-12.61 & 13.365 \\
\hline-12.61 & 13.365 \\
\hline-12.61 & 13.365 \\
\hline-12.550833 & 13.439722 \\
\hline-12.550833 & 13.439722 \\
\hline-12.544166 & 13.4638 \\
\hline
\end{tabular}




\begin{tabular}{|c|c|c|}
\hline Are Lat/Long estimated? & $\begin{array}{c}\text { Original elevation datum } \\
\text { used }\end{array}$ & $\begin{array}{c}\text { Elevation measurement } \\
\text { method }\end{array}$ \\
\hline Yes & $\begin{array}{c}\text { Mean Sea Level / General } \\
\text { definition }\end{array}$ & Not reported \\
\hline Yes & N/A & N/A \\
\hline Yes & N/A & N/A \\
\hline Yes & $\begin{array}{c}\text { Mean Sea Level / General } \\
\text { definition }\end{array}$ & $\begin{array}{l}\text { Total station or } \\
\text { Auto/hand level }\end{array}$ \\
\hline Yes & $\begin{array}{c}\text { Mean Sea Level / General } \\
\text { definition }\end{array}$ & $\begin{array}{l}\text { Total station or } \\
\text { Auto/hand level }\end{array}$ \\
\hline Yes & N/A & N/A \\
\hline Yes & N/A & N/A \\
\hline Yes & $\begin{array}{c}\text { Mean Sea Level / General } \\
\text { definition }\end{array}$ & $\begin{array}{l}\text { Total station or } \\
\text { Auto/hand level }\end{array}$ \\
\hline Yes & $\begin{array}{c}\text { Mean Sea Level / General } \\
\text { definition }\end{array}$ & N/A \\
\hline Yes & $\begin{array}{c}\text { Mean Sea Level / General } \\
\text { definition }\end{array}$ & $\begin{array}{l}\text { Total station or } \\
\text { Auto/hand level }\end{array}$ \\
\hline Yes & N/A & N/A \\
\hline & N/A & N/A \\
\hline Yes & N/A & N/A \\
\hline & N/A & N/A \\
\hline Yes & N/A & N/A \\
\hline Yes & N/A & $\mathrm{N} / \mathrm{A}$ \\
\hline
\end{tabular}


Yes

Yes

Yes

Yes

Yes

Yes

Yes

Yes

Yes

Yes

Yes

Yes

Yes

Yes

Yes

Yes
N/A

N/A

N/A

N/A

N/A

N/A

N/A

N/A

N/A

N/A

N/A

N/A

N/A

N/A

N/A

Mean Sea Level / General definition

N/A
N/A

N/A

N/A

N/A

N/A

N/A

N/A

N/A

N/A

N/A

N/A

N/A

N/A

N/A

N/A

N/A

N/A 
Yes

Yes

Yes

Yes

Yes

Yes

Yes

Yes

No

No

No

No

No

No

No
Mean Low Tide (MLT)

Mean Low Tide (MLT)

Mean Low Tide (MLT)

N/A

N/A

$\mathrm{N} / \mathrm{A}$

$\mathrm{N} / \mathrm{A}$

Mean Sea Level / General definition

N/A

N/A

N/A

N/A

N/A

N/A

N/A
Total station or

Auto/hand level

Total station or

Auto/hand level

Total station or

Auto/hand level

N/A

N/A

$\mathrm{N} / \mathrm{A}$

N/A

Not reported

N/A

N/A

N/A

$\mathrm{N} / \mathrm{A}$

N/A

N/A

N/A 


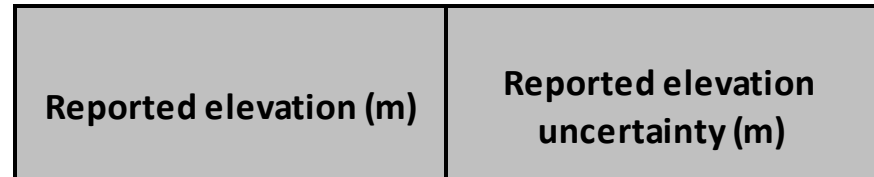

Elevation above MSL (m)

6.5

1

N/A

$-15$

1

N/A

$-16$

1

N/A

6.5

N/A

6.5

4.5

N/A

4.5

9

N/A

N/A

5.6

N/A

N/A

0.5

0.1

N/A

11.7

0.1

11.7

6.5

N/A

N/A

10.5

0.1

N/A

$-33$

N/A

N/A

$-30$

N/A

N/A

0

N/A

N/A

$-34$

N/A

N/A

$-30$

N/A

N/A 
9

0.05

N/A

9

0.05

N/A

6

0.05

N/A

3.5

0.05

N/A

20.2

0.05

N/A

13.3

0.05

N/A

10.4

0.05

N/A

9.5

0.05

N/A

4.5

N/A

N/A

$2.5-6$

N/A

N/A 


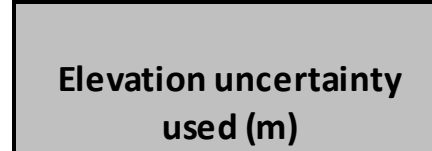

N/A

N/A

N/A

N/A

N/A

N/A

N/A

N/A

N/A

N/A

N/A

N/A

N/A

N/A

N/A

N/A
Age is

Older/Equal/Younger than

Equal to

Equal to

Equal to

Equal to

Equal to

Equal to

Equal to

Equal to

Equal to

Equal to

Equal to

Equal to

Equal to

Equal to

Equal to

Equal to 
N/A

N/A

N/A

N/A

N/A

N/A

N/A

N/A

N/A

N/A

N/A

N/A

N/A

N/A

N/A

N/A

N/A
Equal to

Equal to

Equal to

Equal to

Equal to

Equal to

Equal to

Equal to

Equal to

Equal to

Equal to

Equal to

Equal to

Equal to

Equal to

Equal to

Equal to 
Levelled to Orthometric

N/A zero=Land levelling datum=WGS84

Equal to

Levelled to Orthometric

N/A zero=Land levelling datum=WGS84

Equal to Levelled to Orthometric

N/A zero=Land levelling datum=WGS84

Equal to

N/A

Equal to

N/A

Equal to

N/A

Equal to

N/A

Equal to

N/A

Equal to

N/A

Equal to

N/A

Equal to

N/A

Equal to

N/A

Equal to

N/A

Equal to

N/A

Equal to

N/A

Equal to 


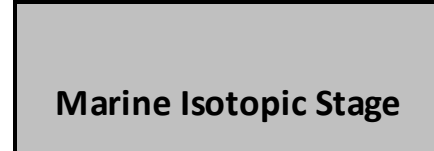

Comments/details on MIS designation or age
Age (ka)

118

127

93

127

MIS 5

130

MIS 5

125

MIS 5

118

MIS 5

138

MIS 5

lowermost part of tidal inlet unit

MIS 5

113

MIS 5

122

MIS 5

13.8

N/A

125

N/A

122

N/A

N/A

142

N/A 
N/A

122

N/A

117

N/A

115

N/A

103

N/A

87

N/A

119.7

N/A

118

N/A

124.3

N/A

116.3

N/A

111.2

N/A

116.1

MIS 5

123.4

MIS 5

135.4

MIS 5

128.4

MIS 5

128.1

MIS 5

124.8

MIS 5

117.3 
MIS 5

115.8

N/A

125

N/A

116

N/A

125

N/A

118

N/A

228

N/A

107

N/A

107

N/A

119

MIS 3

57.8

MIS 3

36.8

MIS 3

47

N/A

53.9

N/A

42.5

N/A

46.8

N/A

44.7 


\begin{tabular}{|c|c|c|}
\hline Age uncertainty (ka) & Aliquot size & $\begin{array}{c}\text { Equivalent Dose } \\
\text { measurement protocol }\end{array}$ \\
\hline
\end{tabular}

7

7

6

5.7

8.2

6.7

7.2

7.3

5.1

5.6

6.9

1.4

12

15

12 
8.4

8.6

9.2

7.5

7.3

6.9

7.9

9.2

8.4

5.2

6.2 
9

9

9

18

25

7

7

9

11.2

3.4

5.9

6.2

3.7

7.9

5.6 


\begin{tabular}{|c|c|c|}
\hline $\begin{array}{c}\text { Treatment during } \\
\text { measurement }\end{array}$ & $\begin{array}{c}\text { Statistical model for } \\
\text { burial dose }\end{array}$ & Machine type \\
\hline
\end{tabular}






\begin{tabular}{|c|c|c|}
\hline Detection unit & Stimulation unit & $\begin{array}{c}\text { Optical Filters used for } \\
\text { Detection }\end{array}$ \\
\hline
\end{tabular}






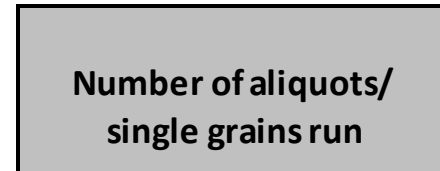

Number of aliquots/ single grains used in sample

N/A

N/A

undefined

N/A

N/A

undefined

N/A

N/A

undefined

N/A

N/A

undefined

N/A

N/A

undefined

N/A

N/A

undefined

N/A

N/A

N/A

N/A

N/A

N/A

N/A

N/A

N/A

N/A
N/A

undefined

N/A

N/A

N/A

N/A

N/A

N/A

N/A

N/A

N/A

undefined 
N/A

N/A

undefined

N/A

N/A

undefined

N/A

N/A

undefined

N/A

N/A

undefined

N/A

N/A

undefined

N/A

N/A

undefined

N/A

N/A

undefined

N/A

N/A

undefined

N/A

N/A

undefined

N/A

N/A

undefined

N/A

N/A

undefined

N/A

N/A

undefined

N/A

N/A

undefined

N/A

N/A

undefined

N/A

N/A

undefined

N/A

N/A

undefined

N/A

N/A

undefined 
N/A

N/A

N/A

N/A

N/A

N/A

N/A

N/A

N/A

N/A

N/A

N/A

N/A

N/A

N/A

N/A
N/A

N/A

N/A

N/A

N/A

N/A

N/A

N/A

N/A

N/A

N/A

N/A

N/A

N/A

N/A

N/A undefined

undefined

undefined

undefined

undefined

undefined

undefined

undefined

undefined

undefined

undefined

undefined

undefined

undefined

undefined

undefined 


\begin{tabular}{|l|l|l|}
\hline Equivalent dose (Gy) & $\begin{array}{l}\text { Equivalent dose } \\
\text { uncertainty (Gy) }\end{array}$ & Overdispersion value (\%) \\
\hline
\end{tabular}






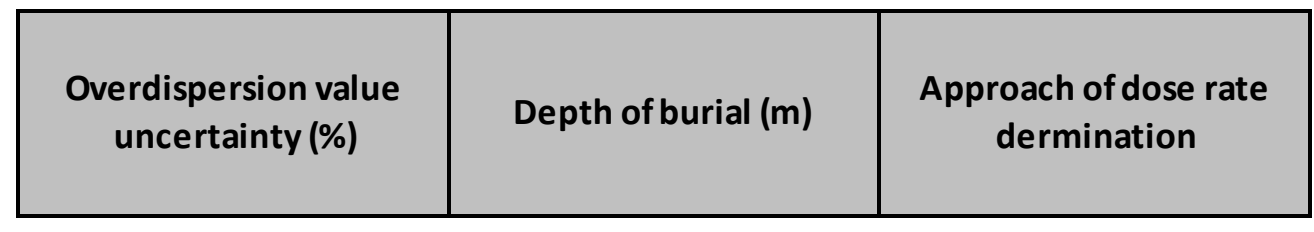

N/A

N/A

N/A

N/A

N/A

N/A

N/A

N/A

N/A

N/A

N/A

N/A

N/A

N/A

N/A

N/A 
N/A

N/A

N/A

N/A

N/A

N/A

N/A

N/A

N/A

N/A

N/A

N/A

N/A

N/A

N/A

N/A

N/A 
N/A

N/A

N/A

N/A

N/A

N/A

N/A

N/A

N/A

N/A

N/A

N/A

N/A

N/A

N/A

N/A 


\begin{tabular}{|c|l|l|}
\hline Uncertainty levels & Uranium content (ppm) & $\begin{array}{c}\text { Uranium content } \\
\text { uncertainty (ppm) }\end{array}$ \\
\hline
\end{tabular}






\begin{tabular}{|l|l|l|}
\hline Thorium content (ppm) & $\begin{array}{c}\text { Thorium content } \\
\text { uncertainty (ppm) }\end{array}$ & Potassium content (\%) \\
\hline
\end{tabular}






\begin{tabular}{|c|l|l|}
\hline $\begin{array}{c}\text { Potassium content } \\
\text { uncertainty (\%) }\end{array}$ & Rubidium content (ppm) & $\begin{array}{l}\text { Rubidium content } \\
\text { uncertainty (ppm) }\end{array}$ \\
\hline
\end{tabular}






\begin{tabular}{|l|l|l|}
\hline Alpha dose rate (Gy/ka) & $\begin{array}{c}\text { Alpha dose rate } \\
\text { uncertainty (Gy/ka) }\end{array}$ & Gamma dose rate (Gy/ka) \\
\hline
\end{tabular}






\begin{tabular}{|c|c|c|}
\hline $\begin{array}{c}\text { Gamma dose rate } \\
\text { uncertainty (Gy/ka) }\end{array}$ & Beta dose rate (Gy/ka) & $\begin{array}{c}\text { Beta dose rate } \\
\text { uncertainty (Gy/ka) }\end{array}$ \\
\hline
\end{tabular}






\begin{tabular}{|c|c|c|}
\hline Cosmic dose rate (Gy/ka) & $\begin{array}{c}\text { Cosmic dose rate } \\
\text { uncertainty (Gy/ka) }\end{array}$ & $\begin{array}{c}\text { Field water content (\% of } \\
\text { dry mass) }\end{array}$ \\
\hline
\end{tabular}






\begin{tabular}{|c|c|c|}
\hline $\begin{array}{c}\text { Water content } \\
\text { determination method }\end{array}$ & $\begin{array}{c}\text { Water Content used in } \\
\text { Final Age Calculation (\% } \\
\text { of dry mass) }\end{array}$ & $\begin{array}{c}\text { Water Content used in } \\
\text { Final Age Calculation } \\
\text { uncertainty (\% of dry } \\
\text { mass) }\end{array}$ \\
\hline
\end{tabular}






\begin{tabular}{|l|l|l|}
\hline Alpha attenuation factor & $\begin{array}{c}\text { Dose Rate conversion } \\
\text { factors }\end{array}$ & Total dose rate (Gy/Ka) \\
\hline
\end{tabular}






\begin{tabular}{|c|c|c|}
\hline $\begin{array}{c}\text { Total dose rate } \\
\text { uncertainty (Gy/ka) }\end{array}$ & $\begin{array}{c}\text { Was fading correction } \\
\text { applied? }\end{array}$ & $\begin{array}{c}\text { Method of fading } \\
\text { correction }\end{array}$ \\
\hline
\end{tabular}






\begin{tabular}{|l|l|l|}
\hline g-value (\% per decade) & $\begin{array}{c}\text { g-value }( \pm 1 \sigma)(\% \text { per } \\
\text { decade) }\end{array}$ & Comments \\
\hline
\end{tabular}






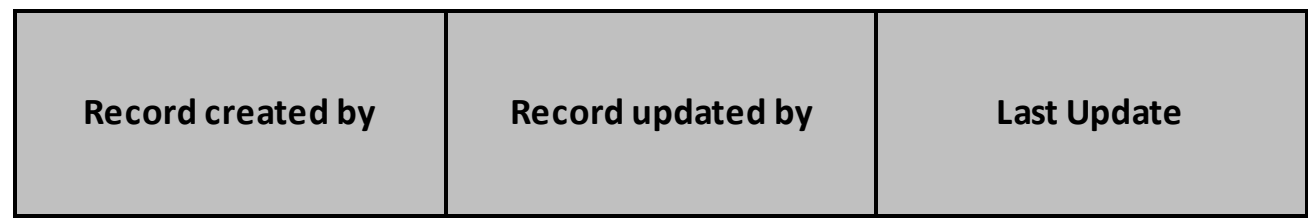

Andrew Cooper

Andrew Cooper

Andrew Cooper

WALIS Admin

2020-12-16 11:10:08

Andrew Cooper

WALIS Admin

2020-12-16 11:10:24

Andrew Cooper

WALIS Admin

2020-12-16 11:11:20

Andrew Cooper

WALIS Admin

2020-12-16 11:11:39

Andrew Cooper

WALIS Admin

2020-12-16 11:11:54

Andrew Cooper

WALIS Admin

2020-12-16 11:12:48

Andrew Cooper

WALIS Admin

2020-12-16 11:13:01

Andrew Cooper

WALIS Admin

2020-12-16 11:13:17

Andrew Cooper

WALIS Admin

2020-12-16 11:13:31

Andrew Cooper

WALIS Admin

2020-12-16 11:13:49

Andrew Cooper

Andrew Cooper

2020-12-16 10:04:39

Andrew Cooper

Andrew Cooper

2020-12-16 10:45:13

Andrew Cooper

Andrew Cooper

2020-12-16 10:45:39

Andrew Cooper

Andrew Cooper

2020-12-16 11:14:45

Andrew Cooper

Andrew Cooper 
Andrew Cooper

Andrew Cooper

Andrew Cooper

Andrew Cooper

Andrew Cooper

Andrew Cooper

Andrew Cooper

Andrew Cooper

Andrew Cooper

Andrew Cooper

Andrew Cooper

Andrew Cooper

Andrew Cooper

Andrew Cooper

Andrew Cooper

Andrew Cooper

Andrew Cooper
Andrew Cooper

2020-12-16 11:15:44

Andrew Cooper

2020-12-16 11:16:19

Andrew Cooper

2020-12-16 11:16:31

Andrew Cooper

2020-12-16 11:16:46

Andrew Cooper

2020-12-16 11:16:57

WALIS Admin

2020-12-16 11:17:31

WALIS Admin

2020-12-16 11:17:46

WALIS Admin

2020-12-16 11:17:59

WALIS Admin

2020-12-16 11:18:10

WALIS Admin

2020-12-16 11:18:57

Andrew Cooper

2020-12-16 11:19:08

Andrew Cooper

2020-12-16 11:19:20

Andrew Cooper

2020-12-16 11:19:31

Andrew Cooper

2020-12-16 11:19:42

Andrew Cooper

2020-12-16 11:20:07

WALIS Admin

2020-12-16 11:21:01

Andrew Cooper 
WALIS Admin

WALIS Admin

WALIS Admin

WALIS Admin

WALIS Admin

WALIS Admin 


\begin{tabular}{|l|l|l|}
\hline WALIS Strat ID & Is this datapoint public? & Chronostratigraphy ID \\
\hline
\end{tabular}

57

1

Isipingo Formation on

Durban Bluff 


\begin{tabular}{|l|c|c|}
\hline Reference(s) & $\begin{array}{c}\text { Description for } \\
\text { chronostratigraphic } \\
\text { constraint }\end{array}$ & Unit thickness (m) \\
\hline
\end{tabular}

Isipingo Formation

Porat and Botha, 2008 aeolianite dated on

Durban Bluff at $182+/-18$ 


\begin{tabular}{|c|c|c|}
\hline $\begin{array}{c}\text { Age is } \\
\text { Older/Equal/Younger } \\
\text { than }\end{array}$ & Marine Isotopic Stage & $\begin{array}{c}\text { Comments/details on } \\
\text { MIS designation }\end{array}$ \\
\hline
\end{tabular}

Equal to

MIS 7 


\begin{tabular}{|l|l|l|}
\hline Upper Age (ka) & Lower Age (ka) & $\begin{array}{c}\text { Notes on age } \\
\text { determination }\end{array}$ \\
\hline
\end{tabular}




\begin{tabular}{|c|c|c|}
\hline $\begin{array}{c}\text { Is this an official } \\
\text { stratigraphic designation? }\end{array}$ & $\begin{array}{c}\text { Has duration estimated in } \\
\text { years? }\end{array}$ & Duration (yrs) \\
\hline
\end{tabular}

Yes 


\begin{tabular}{|c|c|c|}
\hline Duration uncertainty (yrs) & $\begin{array}{c}\text { Is a subzone of a parent } \\
\text { Chronostratigraphy } \\
\text { entry? }\end{array}$ & $\begin{array}{c}\text { Parent record (WALIS } \\
\text { Strat ID) }\end{array}$ \\
\hline
\end{tabular}




\begin{tabular}{|l|l|l|}
\hline Record created by & Record updated by & Last Update \\
\hline
\end{tabular}

Andrew Cooper

Andrew Cooper

2020-04-08 07:28:43 


\begin{tabular}{|c|c|}
\hline $\begin{array}{l}\text { WALIS reference } \\
\text { ID }\end{array}$ & Reference \\
\hline 1330 & Bosman, 2012 \\
\hline 772 & Carr et al., 2010 \\
\hline 1313 & Cawthra 2014 \\
\hline 1315 & Cawthra et al., 2018 \\
\hline 1326 & Cooper and Flores, 1991 \\
\hline 1327 & Cooper and Green, 2016 \\
\hline 1341 & Davies, 1980 \\
\hline 738 & Giresse et al., 1984 \\
\hline 1325 & Hobday, 1975 \\
\hline 769 & Jacobs and Roberts, 2009 \\
\hline 1078 & Kennedy, 2015 \\
\hline 1329 & MR Cooper, 1999 \\
\hline 1075 & Mauz et al., 2015 \\
\hline 1324 & Porat and Botha, 2008 \\
\hline 751 & Ramsay and Cooper, 2002 \\
\hline 1321 & Ramsay et al. 1993 \\
\hline 1296 & Ramsay, 1994 \\
\hline 1316 & Roberts and Berger 1997 \\
\hline 1706 & Roberts, Karkanas et al., 2012 \\
\hline 1068 & Rovere et al., 2016 \\
\hline 1298 & Walker et al., 2016 \\
\hline 1705 & Wang et al., 2008 \\
\hline 621 & Zecchin et al., 2004 \\
\hline
\end{tabular}


Bosman, C., 2012. The marine geol ogy of the Aliwal Shoal, Scottburgh, Carr, A. S., M. D. Bateman, D. L. Roberts, C. V. Murray-Wallace, Z. Jacobs, and P. J. Holmes, 2010, The last interglaci; Research, v. 73 (2), p. 351-363.

Cawthra, H.C., 2014. The marine geology of Mossel Bay, South Africa (Docto

Cawthra, H.C., Jacobs, Z., Compton, J.S., Fisher, E.C., Karkanas, P. and Marean, C.W., 2018. Depositional and sea-lev, South Africa. Quaternary Science Reviews, 181, Cooper, J.A.G., Flores, R.M., 1991. Shoreline deposits and diagenesis resulting from two Late Pleistocene highsta। Cooper, J.A.G. and Green, A.N., 2016. Geomorphology and preservation potential of coastal and submerged aeoliar Davies, O. 1980. Last Interglacial shorelines in the South Cape. Giresse, P., C. T. Hoang, and G. Kouyoumontzakis, 1984, Analysis of vertical movements deduced from a geochrono African Earth Sciences, v. 2 (2), p. 177Hobday, D.K., 1975. Quaternary sedimentation and development of the lagoonal complex, Lake St I Jacobs, Z., and D. L. Roberts, 2009, Last interglacial Age for aeolian and marine deposits and the Nahoon fossil human 160-169.

Kennedy, D.M., 2015. Where is the seaward edge? A review and definition of shore platfor Cooper, M.R., 1999. The Cainozoic palaeontology and stratigraphy of KwaZulu-Natal. Part 3. The Mduku Form Mauz, B., Vacchi, M., Green, A., Hoffmann, G. and Cooper, A., 2015. Beachrock: a tool for reconstruct Porat, N. and Botha, G.A. 2008. The luminescence chronology of dune development on the Maputaland , Ramsay, P. J., and J. A. G. Cooper, 2002, Late Quaternary Sea-Level Change in Soutr Ramsay, P. J., Smith. A. M., Lee-Thorp, J. C., Vogel, J. C., Tyldsley, M., and Kidwell, W. (1993). 130000 year-old fi Science, 89, 165

Ramsay, P.J., 1994. Marine geology of the Sodwana Bay shelf, southeast Afric Roberts, D., and Berger, L. 1997. Last interglacial (c. 117 kyr) human footprints from South $A$ Roberts, D.L., Karkanas, P., Jacobs, Z., Marean, C.W. and Roberts, R.G., 2012. Melting ice sheets 400,000 yr ago rais Letters, 357, 226-237.

Rovere, A., Raymo, M.E., Vacchi, M., Lorscheid, T., Stocchi, P., Gomez-Pujol, L., Harris, D.L., Casella, E., O'Leary, M.J indicators: Reconstructing sea-level in a warmer world. Earth-Sci€

Walker, R.T., Telfer, M., Kahle, R.L., Dee, M.W., Kahle, B., Schwenninger, J.L., Sloan, R.A. and Watts, A.B., 2016. Rar Geoscience, 9(12), pp.909-914.

Wang, Q., Tobias, P., Roberts, D., Jacobs, Z., 2008. A re-examination of a human femur found at the Blind River site, Rev. 71, 43e61.

Zecchin, M., R. Nalin, and C. Roda, 2004, Raised Pleistocene marine terraces of the Crotone peninsula (Calabria, south 172, p. $165-185$. 


\begin{tabular}{|c|c|c|}
\hline Journal & Year & doi \\
\hline \multicolumn{3}{|c|}{2012} \\
\hline Quaternary Research & 2010 & https://doi.org/10.1016/j.yqres.2009.0i \\
\hline \multirow[t]{5}{*}{$\begin{array}{c}\text { Doctoral dissertation, University of } \\
\text { Cape Town }\end{array}$} & 2014 & \\
\hline & 2018 & \\
\hline & 1991 & \\
\hline & 2016 & \\
\hline & 1980 & \\
\hline \multirow[t]{2}{*}{ Journal of African Earth Sciences } & 1984 & https://doi.org/10.1016/S0731-7247/84 \\
\hline & 1975 & \\
\hline Quaternary Geochronology & 2009 & https://doi.org/10.1016/i.quageo.2008. \\
\hline \multirow[t]{2}{*}{ Earth Science Reviews } & 2015 & https://doi.org/10.1016/i.earscirev.201 \\
\hline & 1999 & \\
\hline \multirow[t]{2}{*}{ Marine Geology } & 2015 & https://doi.org/10.1016/i.margeo.2015 \\
\hline & 2008 & \\
\hline \multirow[t]{2}{*}{ Quaternary Research } & 2002 & https://doi.org/10.1006/qres.2001.229 \\
\hline & 1993 & \\
\hline \multirow[t]{3}{*}{ Marine Geology } & 1994 & \\
\hline & 1997 & \\
\hline & 2012 & \\
\hline \multirow[t]{2}{*}{ Earth Science Reviews } & 2016 & https://doi.org/10.1016/i.earscirev.201 \\
\hline & 2016 & \\
\hline Anthropological Review & 2008 & \\
\hline Sedimentary Geology & 2004 & https://doi.org/10.1016/i.sedgeo.2004. \\
\hline
\end{tabular}


8.006

226

https://open.uct.ac.za/handle/11427/81

226

9

4) $80012-9$

226

.09 .002

999

.01 .009

$95,159,62,205$

$\underline{0}$

226

$\underline{6.06 .006}$

999

$\underline{08.003}$

100 


\begin{tabular}{|c|c|}
\hline Language & Timeframe \\
\hline \multicolumn{2}{|l|}{ EN } \\
\hline EN & Pleistocene,MIS 5 \\
\hline \multicolumn{2}{|l|}{ EN } \\
\hline \multicolumn{2}{|l|}{ EN } \\
\hline \multicolumn{2}{|l|}{ EN } \\
\hline \multicolumn{2}{|l|}{ EN } \\
\hline \multicolumn{2}{|l|}{ EN } \\
\hline EN & Holocene,Pleistocene \\
\hline \multicolumn{2}{|l|}{ EN } \\
\hline EN & Pleistocene \\
\hline \multicolumn{2}{|l|}{ EN } \\
\hline \multicolumn{2}{|l|}{ EN } \\
\hline EN & Holocene,Pleistocene \\
\hline \multicolumn{2}{|l|}{ EN } \\
\hline EN & Holocene,Pleistocene,MIS 5 \\
\hline \multicolumn{2}{|l|}{ EN } \\
\hline \multicolumn{2}{|l|}{ EN } \\
\hline \multicolumn{2}{|l|}{ EN } \\
\hline \multicolumn{2}{|l|}{ EN } \\
\hline EN & Pleistocene,MIS 5 \\
\hline \multicolumn{2}{|l|}{ EN } \\
\hline \multicolumn{2}{|l|}{ EN } \\
\hline EN & Pleistocene \\
\hline
\end{tabular}




\begin{tabular}{|c|c|}
\hline Record created by & Record updated by \\
\hline Andrew Cooper & \\
Deirdre Ryan & Ann-Kathrin Petersen \\
Andrew Cooper & WALIS Admin \\
Andrew Cooper & Andrew Cooper \\
Andrew Cooper & \\
Andrew Cooper & Andrew Cooper \\
Andrew Cooper & \\
Deirdre Ryan & Ann-Kathrin Petersen \\
Andrew Cooper & \\
Deirdre Ryan & Ann-Kathrin Petersen \\
Alessio Rovere & Ann-Kathrin Petersen \\
Andrew Cooper & Ann-Kathrin Petersen \\
Alessio Rovere & Andrew Cooper \\
Andrew Cooper & Ann-Kathrin Petersen \\
Deirdre Ryan & Andrew Cooper \\
Andrew Cooper & \\
Andrew Cooper & \\
Andrew Cooper & \\
Andrew Cooper & \\
Alessio Rovere & \\
Andrew Cooper & \\
Andre Ryan & \\
\hline
\end{tabular}




\begin{tabular}{|l|l|}
\hline Last Update & Type of paper \\
\hline
\end{tabular}

2020-04-08 10:54:08

2019-07-23 14:11:25

2020-12-16 10:28:27

2020-04-06 10:38:08

2020-04-08 06:42:42

2020-04-08 09:27:42

2020-04-20 08:20:21

2019-07-23 08:23:26

2020-04-08 06:41:39

2019-07-23 14:07:36

2019-07-28 05:08:33

Review

2020-04-08 10:30:42

2019-07-28 05:01:23

Review

2020-04-08 06:37:58

2019-07-23 08:52:13

2020-04-07 08:43:33

2020-04-02 10:58:47

2020-04-06 10:48:23

2020-11-26 17:01:22

2019-07-28 04:15:46

2020-04-03 09:31:00

2020-11-26 13:03:52

2019-07-22 11:29:56

RSL indicators source 